\title{
Inelastic electron scattering from the three-nucleon bound states with polarization
}

\author{
R.-W. Schulze ${ }^{1}$ and P. U. Sauer ${ }^{1,2}$ \\ ${ }^{1}$ Theoretical Physics, University Hannover, 3000 Hannover, Germany \\ ${ }^{2}$ Department of Physics, University of Washington, Seattle, Washington 98195 \\ (Received 13 July 1992)
}

\begin{abstract}
Spin-dependent inelastic electron scattering from polarized ${ }^{3} \mathrm{He}$ is studied. The theoretical description uses the plane-wave impulse approximation. A spin-dependent spectral function, which summarizes all of the nuclear structure information on the process, is introduced. The formalism is developed for inclusive processes in all kinematic regimes of inelastic electron scattering. A comparison between theoretical predictions and experimental data is carried out in the region of quasifree scattering. The use of a polarized ${ }^{3} \mathrm{He}$ target as a neutron spin target is discussed.
\end{abstract}

PACS number(s): 25.30. $-\mathrm{c}, 24.70 .+\mathrm{s}, 25.10 .+\mathrm{s}, 29.25 . \mathrm{Pj}$

\section{INTRODUCTION}

Inelastic electron scattering from the three-nucleon bound states ${ }^{3} \mathrm{He}$ and ${ }^{3} \mathrm{H}$ attracts special experimental and theoretical attention. Data for inclusive processes cover the kinematic regimes of quasielastic scattering $[1-3]$, quasifree pion production [1], and deep-inelastic $[3,4]$ scattering. They refer to unpolarized electron beams and spin-averaged targets. The theoretical description [5-8] is usually in the plane-wave impulse approximation (PWIA); the inclusion $[9,10]$ of the finalstate interaction for quasielastic scattering has just begun.

The present paper extends the theoretical PWIA description of Ref. [6] to inclusive processes with polarized electron beams and polarized ${ }^{3} \mathrm{He}$ and ${ }^{3} \mathrm{H}$ targets. A polarized ${ }^{3} \mathrm{He}$ target is especially interesting as a possible substitute for a polarized neutron target [11]. Section II discusses the general properties of the nuclear current tensor with polarization of the target and defines the nuclear structure functions. Section III derives the nuclear current tensor for the three-nucleon bound states in PWIA. The notion of a spin-dependent spectral function is introduced. It is the actual technical backbone of the theoretical description. Its properties and its practical computation are discussed. Examples for ${ }^{3} \mathrm{He}$ spin structure functions are given. Section IV derives the polarized cross sections for inclusive processes. Section V compares theoretical predictions and experimental data for inelastic electron scattering in the kinematic regime of quasielastic scattering. The problem of extracting electromagnetic neutron properties from those experiments is discussed. Section VI contains conclusions with a discussion of possible extensions of the results obtained so far.

\section{GENERAL PROPERTIES OF THE NUCLEAR CURRENT TENSOR}

The current tensor $W_{A}^{\mu \nu}\left(Q, P_{A}\right)$ of a target $A$ with mass $m_{A}$, four-momentum $P_{A}$, and polarization $n_{A}$ is required for the description of inclusive processes; it is defined by

$$
\left\langle n_{A}\left|W_{A}^{\mu \nu}\left(Q, P_{A}\right)\right| n_{A}\right\rangle=(2 \pi)^{6} \frac{P_{A}^{0}}{m_{A}} \sum_{\beta_{X}} \int d^{3} P_{X}\left\langle\mathbf{P}_{A} n_{A}\left|J_{A}^{\mu}(0)\right| \mathbf{P}_{X} \beta_{X}\right\rangle \delta^{4}\left(P_{X}-Q-P_{A}\right)\left\langle\mathbf{P}_{X} \beta_{X}\left|J_{A}^{\nu}(0)\right| \mathbf{P}_{A} n_{A}\right\rangle
$$

in terms of the nuclear current $J_{A}^{\mu}(0)$ at time-space point 0 ; the proton charge $e_{p}$ is split off from the current. General final states $\left|\mathbf{P}_{X} \beta_{X}\right\rangle$ of c.m. momentum $\mathbf{P}_{X}$ can be reached in the scattering process, $\beta_{X}$ describing discrete quantum numbers and also the modes of internal nuclear excitation; they are not observed in inclusive processes. The momentum transfer to the target nucleus is $Q$. The current tensor is Hermitian and conserved, it preserves parity and timereversal invariance. Its Lorentz structure is built from the three four-vectors $Q, P_{A}$, and $n_{A}$ which satisfy $P_{A}^{2}=m_{A}^{2}$, $n_{A}^{2}=-1$, and $\left(P_{A} \cdot n_{A}\right)=0$. The current tensor has the general form

$$
\begin{aligned}
\left\langle n_{A}\left|W_{A}^{\mu \nu}\left(Q, P_{A}\right)\right| n_{A}\right\rangle= & \left(\frac{Q^{\mu} Q^{v}}{Q^{2}}-g^{\mu \nu}\right) W_{1}^{A}\left(Q^{2}, Q \cdot P_{A} / m_{A}\right)+\widetilde{P}_{A}^{\mu} \widetilde{P}_{A}^{v} \frac{W_{2}^{A}\left(Q^{2}, Q \cdot P_{A} / m_{A}\right)}{m_{A}^{2}} \\
& +i \epsilon^{\mu v \alpha \beta} Q_{\alpha}\left(n_{A \beta} \frac{G_{1}^{A}\left(Q^{2}, Q \cdot P_{A} / m_{A}\right)}{m_{A}}+\left[\left(Q \cdot P_{A}\right) n_{A \beta}-\left(Q \cdot n_{A}\right) P_{A \beta}\right] \frac{G_{2}^{A}\left(Q^{2}, Q \cdot P_{A} / m_{A}\right)}{m_{A}^{3}}\right)
\end{aligned}
$$


for a spin- $\frac{1}{2}$ nucleus with

$$
\widetilde{P}_{A}:=P_{A}-\frac{Q \cdot P_{A}}{Q^{2}} Q
$$

In Eq. (2.2a) $g^{\mu \nu}$ is the metric tensor with $g^{00}=-g^{11}=-g^{22}=-g^{33}=1$ and $\epsilon^{\mu \nu \alpha \beta}$ the totally antisymmetric tensor in four dimensions with $\epsilon_{0123}=-\epsilon^{0123}=1$. The notation $\left\langle n_{A}\left|W_{A}^{\mu \nu}\left(Q, P_{A}\right)\right| n_{A}\right\rangle$ indicates that the current tensor $W_{A}^{\mu \nu}\left(Q, P_{A}\right)$ is considered an operator in spin space.

The spin-averaged structure functions $W_{1}^{A}$ and $W_{2}^{A}$ and the spin structure functions $G_{1}^{A}$ and $G_{2}^{A}$ determine the current tensor. In turn, they determine the cross section of the inclusive processes which are described by the current tensor. The structure functions are real-valued Lorentz scalars and therefore depend on the scalars $Q^{2}$ and $\left(Q \cdot P_{A}\right)$. The dependence of the current tensor on the polarization vector $n_{A}$ must be linear for spin- $\frac{1}{2}$ targets; the dependence on the pseudoscalar $\left(Q \cdot n_{A}\right)$ is explicitly split off from the spin structure functions. The structure functions can be obtained from a given current tensor by contraction with other tensors, i.e., the spinaveraged structure functions by

$$
\begin{aligned}
W_{1}^{A}\left(Q^{2}, Q \cdot P_{A} / m_{A}\right) \\
\quad=\frac{1}{2}\left[\frac{\widetilde{P}_{A \mu} \widetilde{P}_{A v}}{\widetilde{P}_{A}^{2}}-g_{\mu \nu}\right)\left\langle n_{A}\left|W_{A}^{\mu \nu}\left(Q, P_{A}\right)\right| n_{A}\right\rangle,
\end{aligned}
$$

$W_{2}^{A}\left(Q^{2}, Q \cdot P_{A} / m_{A}\right)$

$$
=\frac{1}{2} \frac{m_{A}^{2}}{\widetilde{P}_{A}^{2}}\left[3 \frac{\widetilde{P}_{A \mu} \widetilde{P}_{A v}}{\widetilde{P}_{A}^{2}}-g_{\mu \nu}\right]\left\langle n_{A}\left|W_{A}^{\mu \nu}\left(Q, P_{A}\right)\right| n_{A}\right\rangle
$$

and the spin structure functions by

$$
\begin{aligned}
G_{1}^{A}\left(Q^{2}, Q \cdot P_{A} / m_{A}\right) & \\
= & -\frac{i}{2} \frac{m_{A}}{Q \cdot n_{A}}\left[\left(Q \cdot n_{A}\right) P_{A}^{2} Q^{\alpha} n_{A}^{\beta}-\left(Q \cdot P_{A}\right) Q^{\alpha} P_{A}^{\beta}\right] \\
& \times \frac{\epsilon_{\mu v \alpha \beta}\left\langle n_{A}\left|W_{A}^{\mu \nu}\left(Q, P_{A}\right)\right| n_{A}\right\rangle}{P_{A}^{2}\left(Q^{2}+\left(Q \cdot n_{A}\right)^{2}\right)-\left(Q \cdot P_{A}\right)^{2}} \\
G_{2}^{A}\left(Q^{2}, Q \cdot P_{A} / m_{A}\right) & \left.-\left[Q^{2}+\left(Q \cdot n_{A}\right)^{2}\right] Q^{\alpha} P_{A}^{\beta}\right\} \\
= & \frac{i}{2} \frac{m_{A}^{3}}{Q^{2}\left(Q \cdot n_{A}\right)}\left\{\left(Q \cdot n_{A}\right)\left(Q \cdot P_{A}\right) Q^{\alpha} n_{A}^{\beta}\right. \\
& \times \frac{\epsilon_{\mu v \alpha \beta}\left\langle n_{A}\left|W_{A}^{\mu \nu}\left(Q, P_{A}\right)\right| n_{A}\right\rangle}{P_{A}^{2}\left(Q^{2}+\left(Q \cdot n_{A}\right)^{2}\right)-\left(Q \cdot P_{A}\right)^{2}} .
\end{aligned}
$$

The derivation of Eq. (2.3) exploits the identity $\epsilon_{\mu \nu \alpha \beta} \epsilon^{\mu \nu \gamma \rho}=-2\left(g_{\alpha}{ }^{\gamma} g_{\beta}{ }^{\rho}-g_{\alpha}{ }^{\rho} g_{\beta}{ }^{\gamma}\right)$. Current conservation $Q_{\mu} W_{A}^{\mu \nu}\left(Q, P_{A}\right)=W_{A}^{\mu \nu}\left(Q, P_{A}\right) Q_{v}=0$ will be used when evaluating Eqs. (2.3). The result (2.3) for the spin structure functions appears to introduce a dependence on the polarization vector $n_{A}$. That dependence is fake and disappears on further analysis.

Without loss of generality, the nuclear center of mass (c.m.) system is chosen such that the three four-momenta which build up the Lorentz structure of the nuclear current tensor become

$$
\begin{aligned}
& P_{A}=\left(m_{A}, 0\right), \\
& n_{A}=\left(0, n_{A}^{1}, 0, n_{A}^{3}\right), \\
& Q=\left(Q^{0}, 0,0,|\mathbf{Q}|\right) .
\end{aligned}
$$

The polarization vector $n_{A}$ is spacelike and does not have a time component in the c.m. frame, since $\left(P_{A} \cdot n_{A}\right)=0$. The unit vectors $\widehat{\mathbf{e}}_{i}$ of that frame are defined by $\hat{\mathbf{e}}_{3}=\widehat{\mathbf{Q}}$, $\widehat{\mathbf{e}}_{2}=\left(\widehat{\mathbf{e}}_{3} \times \widehat{\mathbf{n}}_{A}\right) /\left|\widehat{\mathbf{e}}_{3} \times \widehat{\mathbf{n}}_{A}\right|$, and $\widehat{\mathbf{e}}_{1}=\widehat{\mathbf{e}}_{2} \times \widehat{\mathbf{e}}_{3}$. In that nuclear c.m. system the structure functions of Eqs. (2.3) take the particular forms

$$
\begin{aligned}
& W_{1}^{A}\left(Q^{2}, Q \cdot P_{A} / m_{A}\right)=\frac{1}{2}\left\langle n_{A}\left|W_{A}^{11}\left(Q, P_{A}\right)+W_{A}^{22}\left(Q, P_{A}\right)\right| n_{A}\right\rangle, \\
& W_{2}^{A}\left(Q^{2}, Q \cdot P_{A} / m_{A}\right)=\frac{Q^{4}}{Q^{4}}\left\langle n_{A}\left|W_{A}^{00}\left(Q, P_{A}\right)\right| n_{A}\right\rangle-\frac{1}{2} \frac{Q^{2}}{Q^{2}}\left\langle n_{A}\left|W_{A}^{11}\left(Q, P_{A}\right)+W_{A}^{22}\left(Q, P_{A}\right)\right| n_{A}\right\rangle, \\
& G_{1}^{A}\left(Q^{2}, Q \cdot P_{A} / m_{A}\right)=-\frac{i}{2} \frac{m_{A}^{2}}{\mathbf{Q}^{2}}\left[\frac{1}{n_{A}^{1}} \frac{Q^{2}}{m_{A}|\mathbf{Q}|}\left\langle n_{A}\left|W_{A}^{02}\left(Q, P_{A}\right)-W_{A}^{20}\left(Q, P_{A}\right)\right| n_{A}\right\rangle\right] \\
& \left.+\frac{1}{n_{A}^{3}} \frac{Q^{0}}{m_{A}}\left\langle n_{A}\left|W_{A}^{12}\left(Q, P_{A}\right)-W_{A}^{21}\left(Q, P_{A}\right)\right| n_{A}\right\rangle\right] \\
& G_{2}^{A}\left(Q^{2}, Q \cdot P_{A} / m_{A}\right)=\frac{i}{2} \frac{m_{A}^{2}}{\mathbf{Q}^{2}}\left\{\frac{1}{n_{A}^{1}} \frac{Q^{0}}{|\mathbf{Q}|}\left\langle n_{A}\left|W_{A}^{02}\left(Q, P_{A}\right)-W_{A}^{20}\left(Q, P_{A}\right)\right| n_{A}\right\rangle\right. \\
& \left.+\frac{1}{n_{A}^{3}}\left\langle n_{A}\left|W_{A}^{12}\left(Q, P_{A}\right)-W_{A}^{21}\left(Q, P_{A}\right)\right| n_{A}\right\rangle\right] \text {. }
\end{aligned}
$$


In the results of Eq. (2.5) all nuclear tensor matrix elements involving the third component of the current are eliminated in favor of matrix elements involving the charge density by current conservation, i.e., by $W_{A}^{3 v}\left(Q, P_{A}\right)=Q^{0} W_{A}^{0 v}\left(Q, P_{A}\right) /|\mathbf{Q}|$ and by $W_{A}^{\mu 3}\left(Q, P_{A}\right)$ $=W_{A}^{\mu 0}\left(Q, P_{A}\right) Q^{0} /|\mathbf{Q}|$. In the c.m. system the structure functions are related to the frame-dependent nuclear response functions, i.e., the spin-averaged transverse and longitudinal ones $R_{T}$ and $R_{L}$, and the spin-dependent transverse and mixed transverse-longitudinal ones $R_{T^{\prime}}$ and $R_{T L}$, by

$$
\begin{aligned}
& W_{1}^{A}\left(Q^{2}, Q \cdot P_{A} / m_{A}\right)=\frac{1}{2} R_{T}\left(Q^{2}, Q^{0}\right), \\
& W_{2}^{A}\left(Q^{2}, Q \cdot P_{A} / m_{A}\right)=\frac{Q^{4}}{Q^{4}} R_{L}\left(Q^{2}, Q^{0}\right) \\
& -\frac{1}{2} \frac{Q^{2}}{\mathbf{Q}^{2}} R_{T}\left(Q^{2}, Q^{0}\right), \\
& G_{1}^{A}\left(Q^{2}, Q \cdot P_{A} / m_{A}\right) \\
& =\frac{1}{2} \frac{m_{A}^{2}}{\mathbf{Q}^{2}} \mid \frac{Q^{2}}{m_{A}|\mathbf{Q}|} \sqrt{\frac{1}{2}} R_{T L^{\prime}}\left(Q^{2}, Q^{0}\right) \\
& \left.+\frac{Q^{0}}{m_{A}} R_{T^{\prime}}\left(Q^{2}, Q^{0}\right)\right] \text {, } \\
& G_{2}^{A}\left(Q^{2}, Q \cdot P_{A} / m_{A}\right) \\
& =-\frac{1}{2} \frac{m_{A}^{2}}{\mathbf{Q}^{2}}\left[\frac{Q^{0}}{|\mathbf{Q}|} \sqrt{\frac{1}{2}} R_{T L^{\prime}}\left(Q^{2}, Q^{0}\right)+R_{T^{\prime}}\left(Q^{2}, Q^{0}\right)\right] \text {. }
\end{aligned}
$$

Those nuclear response functions [12] can directly be computed from the nuclear current tensor according to

$$
R_{T}\left(Q^{2}, Q^{0}\right):=\left\langle n_{A}\left|W_{A}^{11}\left(Q, P_{A}\right)+W_{A}^{22}\left(Q, P_{A}\right)\right| n_{A}\right\rangle,
$$

$R_{L}\left(Q^{2}, Q^{0}\right):=\left\langle n_{A}\left|W_{A}^{00}\left(Q, P_{A}\right)\right| n_{A}\right\rangle$

$$
\begin{aligned}
R_{T^{\prime}}\left(Q^{2}, Q^{0}\right):=-i \frac{1}{n_{A}^{3}}\left\langle n_{A}\right| W_{A}^{12}\left(Q, P_{A}\right) \\
-W_{A}^{21}\left(Q, P_{A}\right)\left|n_{A}\right\rangle,
\end{aligned}
$$

$$
\begin{aligned}
R_{T L^{\prime}}\left(Q^{2}, Q^{0}\right):=-i \sqrt{2} \frac{1}{n_{A}^{1}}\left\langle n_{A}\right| W_{A}^{02}\left(Q, P_{A}\right) \\
-W_{A}^{20}\left(Q, P_{A}\right)\left|n_{A}\right\rangle,
\end{aligned}
$$

or from the structure functions by inverting Eq. (2.6). In fact, we obtain the structure functions numerically by calculating the nuclear response functions according to Eq. (2.7) first. For an energy loss $Q^{0}$ small compared with the three-momentum transfer $|\mathbf{Q}|$ we note that $G_{1}^{A}$ is dominantly determined by $R_{T L}$, whereas $G_{2}^{A}$ is dominantly determined by $R_{T^{\prime}}$. That fact will be demonstrated later on in Figs. 3-6.

\section{THE NUCLEAR CURRENT TENSOR IN PWIA}

The nuclear current tensor is calculated in PWIA as an operator in nuclear spin space, i.e.,

$$
\begin{aligned}
\left\langle s_{A}^{\prime}\left|W_{A}^{\mu \nu}\left(Q, P_{A}\right)\right| s_{A}\right\rangle & \\
=\sum_{t_{N}} \sum_{s_{N} s_{N}^{\prime}} \int d^{3} p_{N} \frac{m_{N}}{p_{N}^{0}} \int & d E\left\langle s_{N}^{\prime}\left|W_{N\left(t_{N}\right)}^{\mu \nu}\left(Q_{N}, p_{N}\right)\right| s_{N}\right\rangle \\
& \times\left\langle s_{N} s_{A}^{\prime}\left|S\left(\mathbf{p}_{N} E t_{N}\right)\right| s_{N}^{\prime} s_{A}\right\rangle .
\end{aligned}
$$

The derivation follows Ref. [6] and is given in Appendix A. It is carried out in the nuclear c.m. system defined in Eq. (2.4). PWIA approximates the complicated $A$ baryon final states $\left|\mathbf{P}_{\mathbf{X}} \beta_{\mathbf{X}}\right\rangle$, which arise in the definition (2.1) of the nuclear current tensor, as a tensor product of single-nucleon states reached through one-nucleon currents from a bound nucleon and of correlated states $\left|\mathbf{P}_{A-1} s_{A-1} f_{A-1}\right\rangle$ in the residual $(A-1)$ nucleus. The residual nucleus is assumed not to participate in the scattering process. Its correlated states $\left|\mathbf{P}_{A-1} s_{A-1} f_{A-1}\right\rangle$ are specified by the momentum $\mathbf{P}_{A-1}$ and the spin quantum number $s_{A-1}$, with $f_{A-1}$ describing the remaining discrete quantum numbers and the modes of internal nuclear excitation. E.g., in the threenucleon system the correlated states of the $(A-1) \mathrm{nu}-$ cleus are the states of the deuteron and of two-nucleon scattering. Due to that approximation on final states, PWIA relates the nuclear current tensor $W_{A}^{\mu \nu}\left(Q, P_{A}\right)$ to that of the single nucleon $W_{N\left(t_{N}\right)}^{\mu \nu}\left(Q_{N}, p_{N}\right)$, which will be discussed in Sec. III A. The kinematics of the scattering process is illustrated in Fig. 1 for PWIA. The fourmomentum of the struck nucleon is $p_{N}=\left(\sqrt{m_{N}^{2}+\mathbf{p}_{N}^{2}}, \mathbf{p}_{N}\right)$; the choice of the time component as $\sqrt{m_{N}^{2}+\mathbf{p}_{N}^{2}}$ is determined by our use of Schrödinger quantum mechanics for nuclear structure; however, other choices and off-mass-shell choices are possible. The nucleonic spin (isospin) projection is denoted by $s_{N}\left(t_{N}\right) ; Q_{N}$ is the momentum transfer to the nucleon by the electron and is different from the momentum transfer $Q$ to the nucleus. The nuclear structure part of $W_{A}^{\mu \nu}\left(Q, P_{A}\right)$ in PWIA is contained in the spectral function $S\left(\mathrm{p}_{N} E t_{N}\right)$. It will be discussed in Sec. III B. Since it is calculated in the

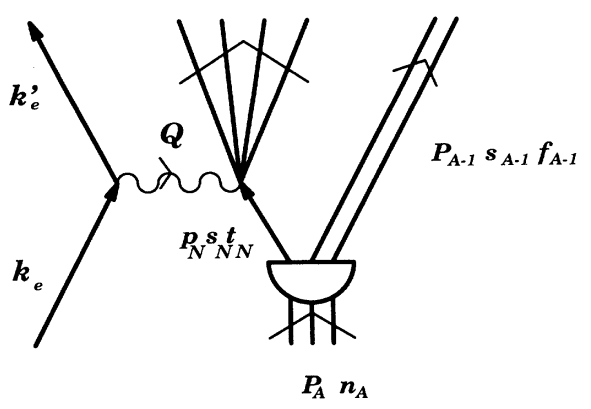

FIG. 1. Inelastic electron scattering from a nucleus in the plane-wave impulse approximation. 
nuclear c.m. system, its dependence on $\mathbf{P}_{A}$ is not notationally indicated. The spin-independent part of $S\left(\mathrm{p}_{N} E t_{N}\right)$ is identical with the usual spectral function of Ref. [5] and is interpreted as the probability for finding a nucleon of isospin $t_{N}$ and of momentum $\mathbf{p}_{N}$ in the target nucleus $A$, the residual $(A-1)$ nucleus having a specified excitation related to the so-called separation energy $E$ according to Eq. (3.4) later on. Both the nucleonic current tensor $W_{N\left(t_{N}\right)}^{\mu \nu}\left(Q_{N}, p_{N}\right)$ and the spectral function $S\left(\mathbf{p}_{N} E t_{N}\right)$ are considered operators in spin space. The quantization axes of the nuclear spin $\sigma_{A}$ and of the nucleonic spin $\sigma_{N}$ are chosen along the $z$ direction for the matrix elements of Eq. (3.1). Thus, general matrix elements, off diagonal with respect to spin, have to be considered when describing scattering from an arbitrarily polarized target. That fact explains the difference in notation between Eqs. (3.1) and (2.1); it is further discussed in Sec. III C.

\section{A. The nucleonic current tensor}

The nucleonic current tensor has the form

$$
\begin{aligned}
W_{N\left(t_{N}\right)}^{\mu \nu}\left(Q_{N}, p_{N}\right)= & {\left[\frac{Q_{N}^{\mu} Q_{N}^{v}}{Q_{N}^{2}}-g^{\mu \nu}\right) W_{1}^{N\left(t_{N}\right)}\left(Q_{N}^{2}, Q_{N} \cdot p_{N} / m_{N}\right)+\widetilde{p}_{N}^{\mu} \widetilde{p}_{N}^{v} \frac{W_{2}^{N\left(t_{N}\right)}\left(Q_{N}^{2}, Q_{N} \cdot p_{N} / m_{N}\right)}{m_{N}^{2}} } \\
& +i \epsilon^{\mu \nu \alpha \beta} Q_{N \alpha} \int s_{\beta}\left(\sigma_{\mathrm{N}}\right) \frac{G_{1}^{N\left(t_{N}\right)}\left(Q_{N}^{2}, Q_{N} \cdot p_{N} / m_{N}\right)}{m_{N}} \\
& \left.+\left\{\left(Q_{N} \cdot p_{N}\right) s_{\beta}\left(\sigma_{\mathrm{N}}\right)-\left[Q_{N} \cdot s\left(\sigma_{\mathrm{N}}\right)\right] p_{N \beta}\right\} \frac{G_{2}^{N\left(t_{N}\right)}\left(Q_{N}^{2}, Q_{N} \cdot p_{N} / m_{N}\right)}{m_{N}^{3}}\right),
\end{aligned}
$$

with

$\widetilde{p}_{N}:=p_{N}-\frac{Q_{N} \cdot p_{N}}{Q_{N}^{2}} Q_{N}$,

$s\left(\sigma_{\mathrm{N}}\right):=\left[\frac{\mathbf{p}_{N} \cdot \sigma_{\mathbf{N}}}{m_{N}}, \boldsymbol{\sigma}_{\mathrm{N}}+\frac{\mathbf{p}_{N} \cdot \boldsymbol{\sigma}_{\mathrm{N}}}{m_{N}\left(m_{N}+p_{N}^{0}\right)} \mathbf{p}_{N}\right)$.

It is derived in Appendix B. It is written as an operator in spin space, the operator character being provided by the spin- $\frac{1}{2}$ operator $\sigma_{\mathrm{N}}$ boosted to $s\left(\sigma_{\mathrm{N}}\right)$ according to Eq. (3.2c). All its diagonal and nondiagonal spin matrix elements required for the nuclear current tensor (3.1) can be obtained from Eq. (3.2a). The nucleonic current tensor depends on the two spin-averaged structure functions $W_{1}^{N\left(t_{N}\right)}$ and $W_{2}^{N\left(t_{N}\right)}$ and on the two spin structure functions $G_{1}^{N\left(t_{N}\right)}$ and $G_{2}^{N\left(t_{N}\right)}$. For elastic scattering from the nucleon, the structure functions are determined by the elastic Sachs form factors $G_{E}^{N\left(t_{N}\right)}$ and $G_{M}^{N\left(t_{N}\right)}$, respectively, i.e.,

$$
\begin{array}{r}
W_{1}^{N\left(t_{N}\right)}\left(Q_{N}^{2}, Q_{N} \cdot p_{N} / m_{N}\right) \\
=\tau\left[G_{M}^{N\left(t_{N}\right)}\left(Q_{N}^{2}\right)\right]^{2} 2 m_{N} \delta\left(2 Q_{N} \cdot p_{N}+Q_{N}^{2}\right), \\
W_{2}^{N\left(t_{N}\right)}\left(Q_{N}^{2}, Q_{N} \cdot p_{N} / m_{N}\right) \\
=\frac{\left[G_{E}^{N\left(t_{N}\right)}\left(Q_{N}^{2}\right)\right]^{2}+\tau\left[G_{M}^{N\left(t_{N}\right)}\left(Q_{N}^{2}\right)\right]^{2}}{1+\tau} \\
\times 2 m_{N} \delta\left(2 Q_{N} \cdot p_{N}+Q_{N}^{2}\right),
\end{array}
$$

$$
\begin{aligned}
G_{1}^{N\left(t_{N}\right)}\left(Q_{N}^{2}, Q_{N} \cdot p_{N} / m_{N}\right) & \\
= & -\frac{G_{M}^{N\left(t_{N}\right)}\left(Q_{N}^{2}\right)}{2} \frac{G_{E}^{N\left(t_{N}\right)}\left(Q_{N}^{2}\right)+\tau G_{M}^{N\left(t_{N}\right)}\left(Q_{N}^{2}\right)}{1+\tau} \\
& \times 2 m_{N} \delta\left(2 Q_{N} \cdot p_{N}+Q_{N}^{2}\right) \\
G_{2}^{N\left(t_{N}\right)}\left(Q_{N}^{2}, Q_{N} \cdot p_{N} / m_{N}\right) & \\
= & \frac{G_{M}^{N\left(t_{N}\right)}\left(Q_{N}^{2}\right)}{4} \frac{G_{M}^{N\left(t_{N}\right)}\left(Q_{N}^{2}\right)-\tau G_{E}^{N\left(t_{N}\right)}\left(Q_{N}^{2}\right)}{1+\tau} \\
& \times 2 m_{N} \delta\left(2 Q_{N} \cdot p_{N}+Q_{N}^{2}\right)
\end{aligned}
$$

with $\tau=-Q_{N}^{2} / 4 m_{N}^{2}$ being non-negative. For inelastic scattering from the nucleon, the structure functions have to account for experimental data in a corresponding way. That is done in Ref. [6] for the spin-averaged structure functions $W_{1}^{N\left(t_{N}\right)}$ and $W_{2}^{N\left(t_{N}\right)}$ and for the kinematic domains of quasifree pion production and deep-inelastic scattering.

\section{B. The nuclear spectral function}

The spin-dependent matrix elements of the nuclear spectral function are defined by 


$$
\begin{aligned}
\left\langle s_{N} s_{A}^{\prime}\left|S\left(\mathbf{p}_{N} E t_{N}\right)\right| s_{N}^{\prime} s_{A}\right\rangle=A \sum_{s_{A-1} f_{A-1}} & \delta\left(E+E_{A}-e_{A-1}\left(f_{A-1}\right)\right) \\
& \times\left\langle\mathbf{P}_{A} s_{A}^{\prime} \mid \mathbf{p}_{N} s_{N}^{\prime} t_{N}\left(\mathbf{P}_{A}-\mathbf{p}_{N}\right) s_{A-1} f_{A-1}\right\rangle\left\langle\mathbf{p}_{N} s_{N} t_{N}\left(\mathbf{P}_{A}-\mathbf{p}_{N}\right) s_{A-1} f_{A-1} \mid \mathbf{P}_{A} s_{A}\right\rangle .
\end{aligned}
$$

The on-mass-shell energies of the $(A-1)$ - and $A$-nucleon systems are approximated such that the respective fourmomenta read

$$
\begin{aligned}
& P_{A-1} \simeq\left(\sqrt{(A-1)^{2} m_{N}^{2}+\left(\mathbf{P}_{A}-\mathbf{p}_{N}\right)^{2}}+e_{A-1}\left(f_{A-1}\right),\right. \\
& \left.\mathbf{P}_{A}-\mathbf{p}_{N}\right), \\
& P_{A} \simeq\left(\sqrt{A^{2} m_{N}^{2}+\mathbf{P}_{A}^{2}}+E_{A}, \mathbf{P}_{A}\right)
\end{aligned}
$$

$E_{A}$ being the trinucleon bound-state energy and $e_{A-1}\left(f_{A-1}\right)$ the excitation energy of the residual $(A-1)$-nucleon system. The $\delta$ function $\delta\left(E+E_{A}\right.$ $\left.-e_{A-1}\left(f_{A-1}\right)\right)$ is introduced in order to make the spectral function formally independent from the quantum numbers $f_{A-1}$ of excitation in the residual $(A-1) \mathrm{nu}-$ cleus. The spectral function is an operator in the nucleonic and in the nuclear spin space. Furthermore, it depends on the momentum $\mathbf{p}_{N}$, the separation energy $E$, and the isospin $t_{N}$ of the nucleon. Since it is calculated in the nuclear c.m. system, $\mathbf{P}_{A}=0$. In this paper the spectral function is determined for the three-nucleon bound state ${ }^{3} \mathrm{He}$; the corresponding spectral function of ${ }^{3} \mathrm{H}$ can be obtained from that of ${ }^{3} \mathrm{He}$ by symmetry relations without additional calculation. The dependence of the spectral function on the isospin label $t_{A}$ of the nucleus will therefore be suppressed in the notation.

The tensor structure of the nuclear current tensor (3.1) in PWIA is carried by the nucleonic current tensor (3.2a). Thus, the spectral function is a Lorentz scalar. In the nuclear c.m. system it is a scalar with respect to rotations and parity. The spectral function therefore has the general operator form

$$
\begin{array}{r}
S\left(\mathbf{p}_{N} E t_{N}\right)=\frac{1}{2}\left\{f_{0}\left(p_{N} E t_{N}\right)+f_{1}\left(p_{N} E t_{N}\right) \sigma_{N} \cdot \boldsymbol{\sigma}_{A}\right. \\
+f_{2}\left(p_{N} E t_{N}\right)\left[\left(\sigma_{\mathbf{N}} \cdot \hat{\mathbf{p}}_{N}\right)\left(\sigma_{\mathbf{A}} \cdot \hat{\mathbf{p}}_{N}\right)\right. \\
\left.\left.-\frac{1}{3} \sigma_{\mathbf{N}} \cdot \boldsymbol{\sigma}_{\mathbf{A}}\right]\right\}
\end{array}
$$

in spin space. The considered nucleus and the nucleon are both spin $-\frac{1}{2}$ particles. The only nontrivial proper scalars, which can be constructed from the three vectors $\widehat{\mathbf{p}}_{N}, \sigma_{\mathrm{N}}$, and $\sigma_{\mathrm{A}}$ available in the c.m. system and which are at most linear in the spin operators, are $\sigma_{\mathrm{N}} \cdot \sigma_{\mathrm{A}}$ and $\left[\left(\sigma_{\mathbf{N}} \cdot \hat{\mathbf{p}}_{N}\right)\left(\sigma_{\mathbf{A}} \cdot \hat{\mathbf{p}}_{N}\right)-\frac{1}{3} \sigma_{\mathbf{N}} \cdot \sigma_{\mathbf{A}}\right]$. The other possible sca$\operatorname{lars} \sigma_{\mathbf{N}} \cdot \hat{\mathbf{p}}_{N}, \sigma_{\mathrm{A}} \cdot \hat{\mathbf{p}}_{N}$, and $\left(\hat{\mathbf{p}}_{N} \times \sigma_{\mathrm{N}}\right) \cdot \sigma_{\mathrm{A}}$ are of pseudoscalar nature and so do not contribute to the expansion of
Eq. (3.6). Thus, only the spin-averaged contribution $f_{0}\left(p_{N} E t_{N}\right)$ and the two spin-dependent contributions $f_{1}\left(p_{N} E t_{N}\right)$ and $f_{2}\left(p_{N} E t_{N}\right)$ make up the full spectral function $S\left(\mathrm{p}_{N} E t_{N}\right)$ according to Eq. (3.6). They do not depend on the full three-dimensional vector $\mathbf{p}_{N}$, but just on its magnitude $p_{N}=\left|\mathbf{p}_{N}\right|$, not to be confused with the corresponding four-vector for which the same symbol is used.

The spectral function $S\left(\mathbf{p}_{N} E t_{N}\right)$ has a singular part due to its contribution from the deuteron carrying the energy $\delta$ function of Eq. (3.4) and a smooth part due to its contribution from the two-nucleon scattering states for which the energy $\delta$ function is integrated out by the summation over $f_{A-1}$. Thus, the spectral function allows us to differentiate between two-body and three-body final states. The practical procedure for computing the spinindependent and spin-dependent contributions to the spectral function of Eq. (3.6), its isospin dependence, and sum rules are given in Appendix C.

Reference [13] suggests closure as a reliable approximation for the exact spectral function $S\left(\mathrm{p}_{N} E t_{N}\right)$ and uses $e_{A-1}\left(f_{A-1}\right)=0$ as average excitation energy for the residual two-nucleon system. In that approximation the sum on $s_{A-1}$ and $f_{A-1}$ can be carried out in Eq. (3.4) analytically. The approximated spectral function takes the same singular form as for the two-body breakup with a proton-deuteron final state in scattering from ${ }^{3} \mathrm{He}$, i.e., it becomes a product of the $\delta$ function $\delta\left(E+E_{A}\right)$ and of the nucleon density $\rho\left(\mathrm{p}_{N} t_{N}\right)$ according to Eq. (C11) of Appendix C. That approximated spectral function does not distinguish between two-body and three-body final states any longer.

\section{Convolution formulas for structure functions}

The target nucleus of spin $\frac{1}{2}$ is - in its c.m. systemcharacterized by the general polarization vector $\widehat{\mathbf{n}}_{A}$, i.e.,

$$
\begin{aligned}
& \sigma_{\mathrm{A}} \cdot \hat{\mathbf{n}}_{A}\left|n_{A}\right\rangle=\left|n_{A}\right\rangle, \\
& \rho_{A}\left(\hat{\mathbf{n}}_{A}\right)=\frac{1}{2}\left(1+\hat{\mathbf{n}}_{A} \cdot \sigma_{\mathbf{A}}\right) .
\end{aligned}
$$

The polarization state $\left|n_{A}\right\rangle$ is expanded in terms of spin states $\left|s_{A}\right\rangle$ with the $z$ axis as quantization axis, i.e., $\left|n_{A}\right\rangle=\sum_{s_{A}}\left|s_{A}\right\rangle\left\langle s_{A} \mid n_{A}\right\rangle$. The operator $\rho_{A}$ is the corresponding density matrix; it keeps the form (3.7b) with $\widehat{\mathbf{n}}_{A}^{2} \leq 1$, even if the polarization is not realized by a pure state. For the polarization (3.7) the nuclear current tensor takes the form 


$$
\begin{aligned}
& \left\langle n_{A}\left|W_{A}^{\mu \nu}\left(Q, P_{A}\right)\right| n_{A}\right\rangle=\operatorname{Tr}\left[W_{A}^{\mu \nu}\left(Q, P_{A}\right) \rho_{A}\left(\widehat{\mathbf{n}}_{A}\right)\right], \\
& \left\langle n_{A}\left|W_{A}^{\mu \nu}\left(Q, P_{A}\right)\right| n_{A}\right\rangle=\sum_{t_{N}} \int d^{3} p_{N} \frac{m_{N}}{p_{N}^{0}} \int d E\left\{\left[\left[\frac{Q_{N}^{\mu} Q_{N}^{v}}{Q_{N}^{2}}-g^{\mu \nu}\right) W_{1}^{N\left(t_{N}\right)}\left(Q_{N}^{2}, Q_{N} \cdot p_{N} / m_{N}\right)\right.\right. \\
& \left.+\frac{\widetilde{p}_{N}^{\mu} \widetilde{p}_{N}^{v}}{m_{N}^{2}} W_{2}^{N\left(t_{N}\right)}\left(Q_{N}^{2}, Q_{N} \cdot p_{N} / m_{N}\right)\right] f_{0}\left(p_{N} E t_{N}\right) \\
& +i \epsilon^{\mu v \alpha \beta} Q_{N \alpha}\left[\rho_{\beta}\left(\mathbf{p}_{N} E t_{N} \widehat{\mathbf{n}}_{A}\right) \frac{G_{1}^{N\left(t_{N}\right)}\left(Q_{N}^{2}, Q_{N} \cdot p_{N} / m_{N}\right)}{m_{N}}\right. \\
& +\left\{\left(Q_{N} \cdot p_{N}\right) \mathcal{S}_{\beta}\left(\mathbf{p}_{N} E t_{N} \hat{\mathbf{n}}_{A}\right)-\left[Q_{N} \cdot \mathcal{S}\left(\mathbf{p}_{N} E t_{N} \hat{\mathbf{n}}_{A}\right)\right] p_{N \beta}\right\} \\
& \left.\left.\times \frac{G_{2}^{N\left(t_{N}\right)}\left(Q_{N}^{2}, Q_{N} \cdot p_{N} / m_{N}\right)}{m_{N}^{3}}\right)\right\}
\end{aligned}
$$

The result (3.8) for the nuclear current tensor $W_{A}^{\mu v}\left(Q, P_{A}\right)$ is derived from of Eq. (3.1); Eq. (3.1) needs the nucleonic current tensor (3.2) and the spectral function (3.6). For compactness of notation, the four-vector

$$
\begin{aligned}
& \mathcal{S}\left(\mathbf{p}_{N} E t_{N} \hat{\mathbf{n}}_{A}\right)=\operatorname{Tr}\left[s\left(\sigma_{\mathbf{N}}\right) S\left(\mathbf{p}_{N} E t_{N}\right) \rho_{A}\left(\hat{\mathbf{n}}_{A}\right)\right] \\
& \mathcal{S}\left(\mathbf{p}_{N} E t_{N} \hat{\mathbf{n}}_{A}\right)=s\left(\widehat{\mathbf{n}}_{A}\right)\left[f_{1}\left(p_{N} E t_{N}\right)-\frac{1}{3} f_{2}\left(p_{N} E t_{N}\right)\right]+s\left(\widehat{\mathbf{p}}_{N}\right)\left(\hat{\mathbf{n}}_{A} \cdot \hat{\mathbf{p}}_{N}\right) f_{2}\left(p_{N} E t_{N}\right)
\end{aligned}
$$

is introduced which contains all spin-dependent nuclear structure information; it uses the spin operator $s\left(\sigma_{\mathrm{N}}\right)$ of Eq. (3.2c) and the vectors $\widehat{\mathbf{n}}_{A}$ and $\widehat{\mathbf{p}}_{N}$ boosted by the nucleon momentum $\mathbf{p}_{N}$ as $\sigma_{\mathrm{N}}$ in Eq. (3.2c), i.e.,

$$
\begin{aligned}
& s\left(\hat{\mathbf{n}}_{A}\right)=\left[\frac{\mathbf{p}_{N} \cdot \hat{\mathbf{n}}_{A}}{m_{N}}, \hat{\mathbf{n}}_{A}+\frac{\mathbf{p}_{N} \cdot \hat{\mathbf{n}}_{A}}{m_{N}\left(m_{N}+p_{N}^{0}\right)} \mathbf{p}_{N}\right), \\
& s\left(\hat{\mathbf{p}}_{N}\right)=\left[\frac{\left|\mathbf{p}_{N}\right|}{m_{N}}, \hat{\mathbf{p}}_{N} \frac{p_{N}^{0}}{m_{N}}\right) .
\end{aligned}
$$

In contrast to Eq. (3.8a) the $\mathrm{Tr}$ in Eq. (3.9a) refers to nucleonic and nuclear spin summations. In the nucleonic current tensor (3.2) the momentum transfer $Q_{N}=Q+P_{A}-P_{A-1}-p_{N}$ to the nucleon is different from the momentum transfer to the whole nucleus, i.e., it becomes

$$
Q_{N}=\left(Q^{0}+A m_{N}-E-\sqrt{(A-1)^{2} m_{N}^{2}+\mathbf{p}_{N}^{2}}-p_{N}^{0}, \mathbf{Q}\right)
$$

in the c.m. system with $\mathbf{P}_{A}=0$. It depends on the integration variables of Eq. (3.8), i.e., on the separation energy $E$ and on the momentum $\mathbf{p}_{N}$ of the struck nucleon.

The nuclear current tensor $W_{A}^{\mu \nu}\left(Q, P_{A}\right)$ in PWIA does not satisfy current conservation, i.e., $Q_{\mu} W_{A}^{\mu \nu}\left(Q, P_{A}\right) \neq 0$ and $W_{A}^{\mu \nu}\left(Q, P_{A}\right) Q_{v} \neq 0$ for the nuclear current tensor of Eq. (3.8). When calculating the nuclear structure functions $W_{1}^{A}, W_{2}^{A}, G_{1}^{A}$, and $G_{2}^{A}$ we therefore take the PWIA nuclear current tensor of Eq. (3.8) only for those longitudinal matrix elements needed in Eqs. (2.5) after current conservation is already exploited. That procedure is called scheme (A) and is admittedly a recipe; arguments for that recipe and alternatives are given in Appendix D. The following convolution formulas result for the structure functions in scheme (A), i.e.,

$$
\begin{aligned}
W_{1}^{A}\left(Q^{2}, Q \cdot P_{A} / m_{A}\right)=\sum_{t_{N}} \int d^{3} p_{N} \frac{m_{N}}{p_{N}^{0}} \int d E & \mid W_{1}^{N\left(t_{N}\right)}\left(Q_{N}^{2}, Q_{N} \cdot p_{N} / m_{N}\right) \\
& \left.+\frac{W_{2}^{N\left(t_{N}\right)}\left(Q_{N}^{2}, Q_{N} \cdot p_{N} / m_{N}\right)}{2 m_{N}^{2}}\left|\hat{\mathbf{Q}} \times \mathbf{p}_{N}\right|^{2}\right) f_{0}\left(p_{N} E t_{N}\right),
\end{aligned}
$$




$$
\begin{aligned}
& W_{2}^{A}\left(Q^{2}, Q \cdot P_{A} / m_{A}\right)=\sum_{t_{N}} \int d^{3} p_{N} \frac{m_{N}}{p_{N}^{0}} \int d E\left\{\frac{W_{2}^{N\left(t_{N}\right)}\left(Q_{N}^{2}, Q_{N} \cdot p_{N} / m_{N}\right)}{m_{N}^{2}}\left[\frac{Q^{4}}{\mathbf{Q}^{4}}\left[p_{N}^{0}-Q_{N}^{0} \frac{Q_{N} \cdot p_{N}}{Q_{N}^{2}}\right]^{2}-\frac{1}{2} \frac{Q^{2}}{\mathbf{Q}^{2}}\left|\hat{\mathbf{Q}}^{2} \times \mathbf{p}_{N}\right|^{2}\right]\right. \\
& \left.-W_{1}^{N\left(t_{N}\right)}\left(Q_{N}^{2}, Q_{N} \cdot p_{N} / m_{N}\right) \frac{Q^{2}}{Q^{2}}\left[1-\frac{Q^{2}}{Q_{N}^{2}}\right]\right\} f_{0}\left(p_{N} E t_{N}\right) \\
& G_{1}^{A}\left(Q^{2}, Q \cdot P_{A} / m_{A}\right)=\frac{m_{A}^{2}}{\mathbf{Q}^{2}} \sum_{t_{N}} \int d^{3} p_{N} \frac{m_{N}}{p_{N}^{0}} \int d E\left(-\frac{Q^{2}}{m_{A}} \frac{1}{n_{A}^{1}} g^{1 \alpha}+\frac{Q^{0}}{m_{A}} \frac{1}{n_{A}^{3}}\left(Q_{N}^{0} g^{3 \alpha}-|\mathbf{Q}| g^{0 \alpha}\right)\right) \\
& \times\left(\rho_{\alpha}\left(\mathbf{p}_{N} E t_{N} \hat{\mathbf{n}}_{A}\right) \frac{G_{1}^{N}\left(Q_{N}^{2}, Q_{N} \cdot p_{N} / m_{N}\right)}{m_{N}}\right. \\
& +\left\{\left(Q_{N} \cdot p_{N}\right) \mathcal{S}_{\alpha}\left(\mathbf{p}_{N} E t_{N} \widehat{\mathbf{n}}_{A}\right)\right. \\
& \left.\left.-\left[Q_{N} \cdot \mathcal{S}\left(\mathbf{p}_{N} E t_{N} \hat{\mathbf{n}}_{A}\right)\right] p_{N \alpha}\right\} \frac{G_{2}^{N}\left(Q_{N}^{2}, Q_{N} \cdot p_{N} / m_{N}\right)}{m_{N}^{3}}\right], \\
& G_{2}^{A}\left(Q^{2}, Q \cdot P_{A} / m_{A}\right)=-\frac{m_{A}^{2}}{Q^{2}} \sum_{t_{N}} \int d^{3} p_{N} \frac{m_{N}}{p_{N}^{0}} \int d E\left[Q^{0} \frac{1}{n_{A}^{1}} g^{1 \alpha}+\frac{1}{n_{A}^{3}}\left(Q_{N}^{0} g^{3 \alpha}-|\mathbf{Q}| g^{0 \alpha}\right)\right] \\
& \times \int \mathcal{S}_{\alpha}\left(\mathbf{p}_{N} E t_{N} \hat{\mathbf{n}}_{A}\right) \frac{G_{1}^{N}\left(Q_{N}^{2}, Q_{N} \cdot p_{N} / m_{N}\right)}{m_{N}} \\
& +\left\{\left(Q_{N} \cdot p_{N}\right) \mathcal{S}_{\alpha}\left(\mathbf{p}_{N} E t_{N} \widehat{\mathbf{n}}_{A}\right)\right. \\
& \left.\left.-\left[Q_{N} \cdot \mathcal{S}\left(\mathbf{p}_{N} E t_{N} \hat{\mathbf{n}}_{A}\right)\right] p_{N \alpha}\right\} \frac{G_{2}^{N}\left(Q_{N}^{2}, Q_{N} \cdot p_{N} / m_{N}\right)}{m_{N}^{3}}\right] .
\end{aligned}
$$

In the integration on $\mathbf{p}_{N}$ only the terms in $\mathcal{S}\left(\mathbf{p}_{N} E t_{N} \hat{\mathbf{n}}_{A}\right)\left[\mathcal{S}^{0}\left(\mathbf{p}_{N} E t_{N} \widehat{\mathbf{n}}_{A}\right)\right]$ proportional to $\widehat{\mathbf{n}}_{A}\left[n_{A}^{3}\right]$ survive. Thus, the dependence of the spin structure functions $G_{1}^{A}$ and $G_{2}^{A}$ on the polarization vector $\widehat{\mathbf{n}}_{A}$ drops out. This fact is made explicit in Eq. (D2) of Appendix $\mathrm{D}$, which is used in the numerical analysis. Sample results for the nuclear structure functions in the region of quasielastic scattering are given in Fig. 2; the employed nuclear structure model is described in Sec. V. The correspondence between the spin structure functions $G_{1}^{A}$ and $G_{2}^{A}$ and the nuclear responses $R_{T L^{\prime}}$ and $R_{T^{\prime}}$, respectively, is demonstrated in Figs. 3-6.

\section{Theoretical uncertainties}

What is the accuracy of theoretical predictions in PWIA? PWIA is quite successful in describing unpolarized inclusive processes. With respect to the small and therefore possibly sensitive spin-dependent observables, this paper cannot explore the validity of PWIA. We can only give uncertainties arising (i) from approximations in the calculation of the spectral function and (ii) from the inequivalent extraction schemes for structure functions and responses.

(i) Blankleider and Woloshyn [13] use closure approximation when calculating the spin-dependent spectral
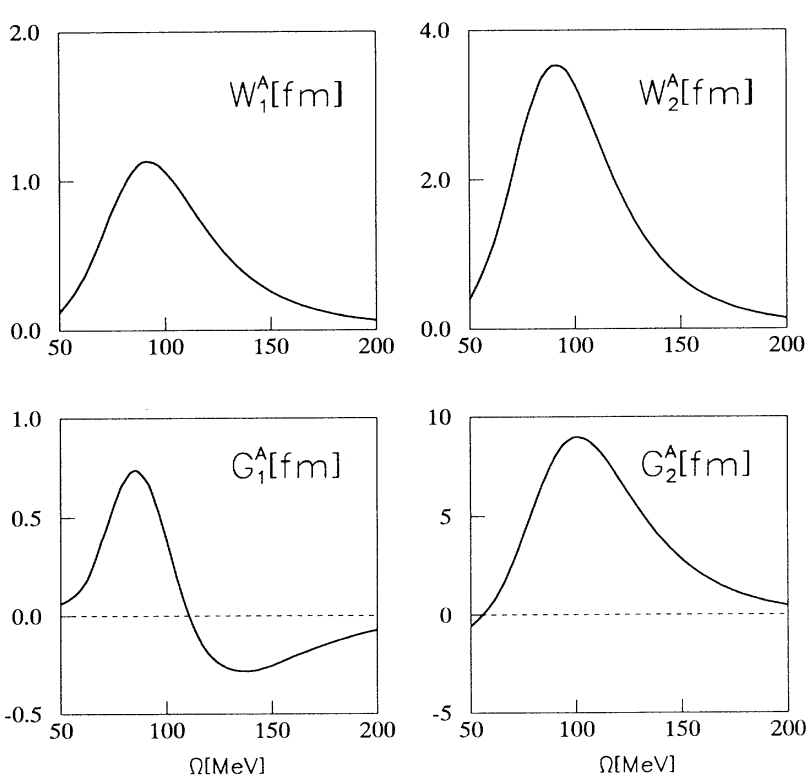

FIG. 2. ${ }^{3} \mathrm{He}$ structure functions $W_{1}^{A}, W_{2}^{A}, G_{1}^{A}$, and $G_{2}^{A}$ as a function of energy loss $\Omega=Q^{0}$ for a four-momentum transfer $Q^{2}=-0.16 \mathrm{GeV}^{2}$. The theoretical result is based on Eqs. (3.11) and (D2). 

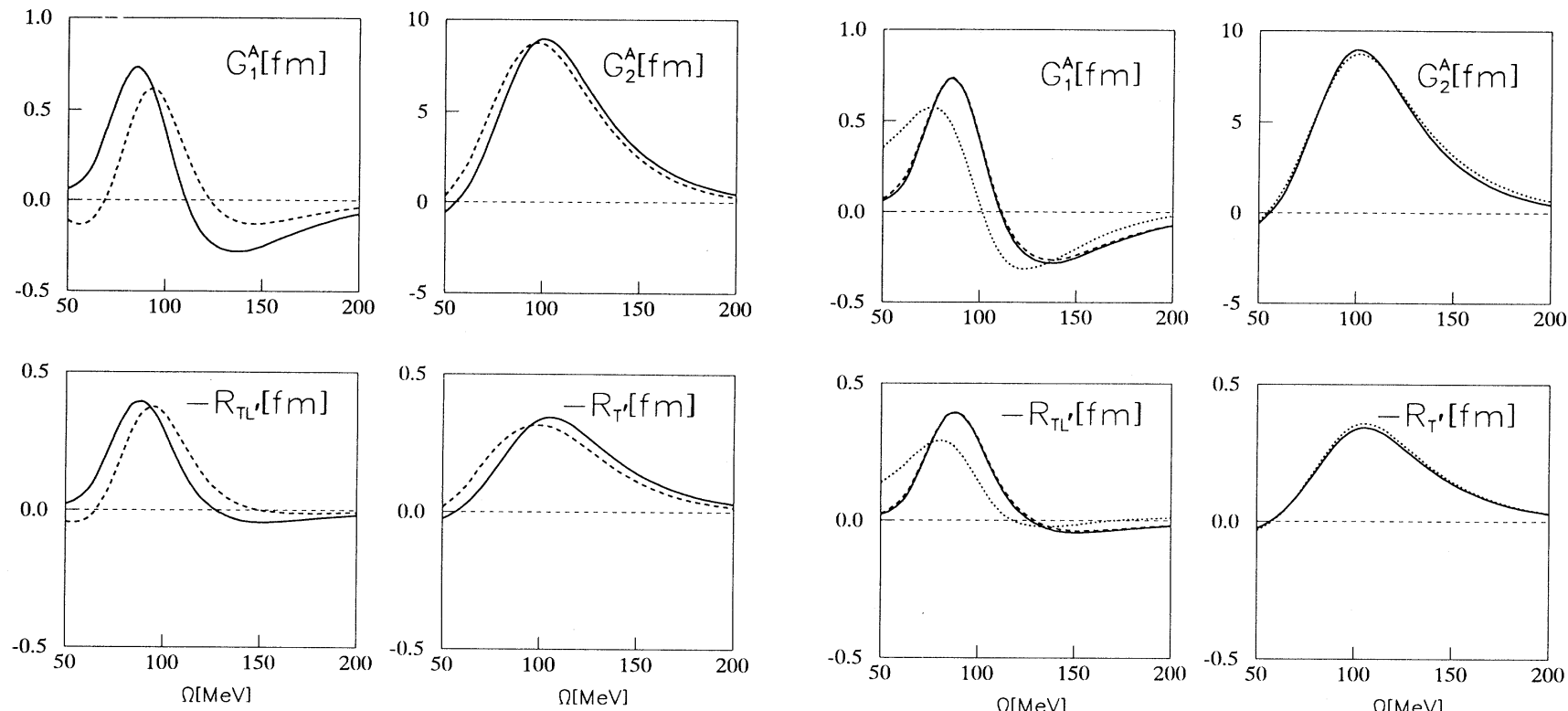

FIG. 3. ${ }^{3} \mathrm{He}$ spin structure functions $G_{1}^{A}$ and $G_{2}^{A}$ and spindependent responses $R_{T L^{\prime}}$ and $R_{T^{\prime}}$ as a function of energy loss $\Omega=Q^{0}$ for a four-momentum transfer $Q^{2}=-0.16 \mathrm{GeV}^{2}$. Compared are results based on the exact spectral function of Eq. (3.4) in Sec. III B (indicated by solid curves) and on the approximated spectral function of Eq. (C11) in Appendix C (indicated by dashed curves). The calculation uses the favored extraction scheme (A) for the spin structure functions and spin-dependent responses according to Eqs. (3.11) and (D2).
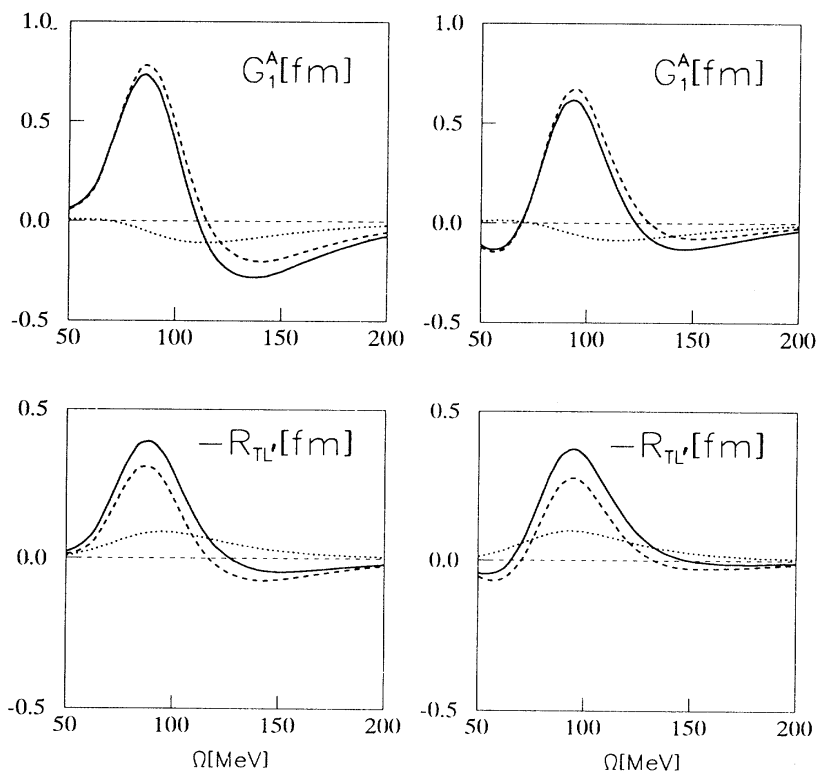

FIG. 4. ${ }^{3} \mathrm{He}$ spin structure function $G_{1}^{A}$ and the spindependent response $R_{T L^{\prime}}$ as a function of energy loss $\Omega=Q^{0}$ for a four-momentum transfer $Q^{2}=-0.16 \mathrm{GeV}^{2}$. Compared are results based on the full spectral function (left side) and closure (right side) using extraction scheme (A) for both. The total result (solid curve) is compared with the respective (dotted curve) and proton (dashed curve) contributions.
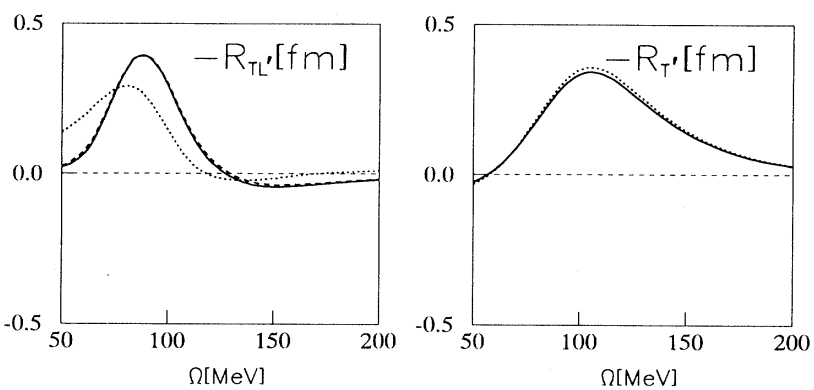

FIG. 5. ${ }^{3} \mathrm{He}$ spin structure functions $G_{1}^{A}$ and $G_{2}^{A}$ and spindependent responses $R_{T L^{\prime}}$ and $R_{T^{\prime}}$ as a function of energy loss $\Omega=Q^{0}$ for a four-momentum transfer $Q^{2}=-0.16 \mathrm{GeV}^{2}$. Compared are results based on the three distinct extraction schemes, i.e., scheme (A) of Eqs. (3.11) and (D2) (indicated by solid curves), scheme (B) of Eq. (D3) (indicated by dashed curves), and scheme (C) of Eq. (D7) (indicated by dotted curves). The unapproximated spectral function of Eq. (3.4) in Sec. III B is used. The solid and dashed curves are indistinguishable for $G_{2}^{A}$ and $\boldsymbol{R}_{T^{\prime}}$
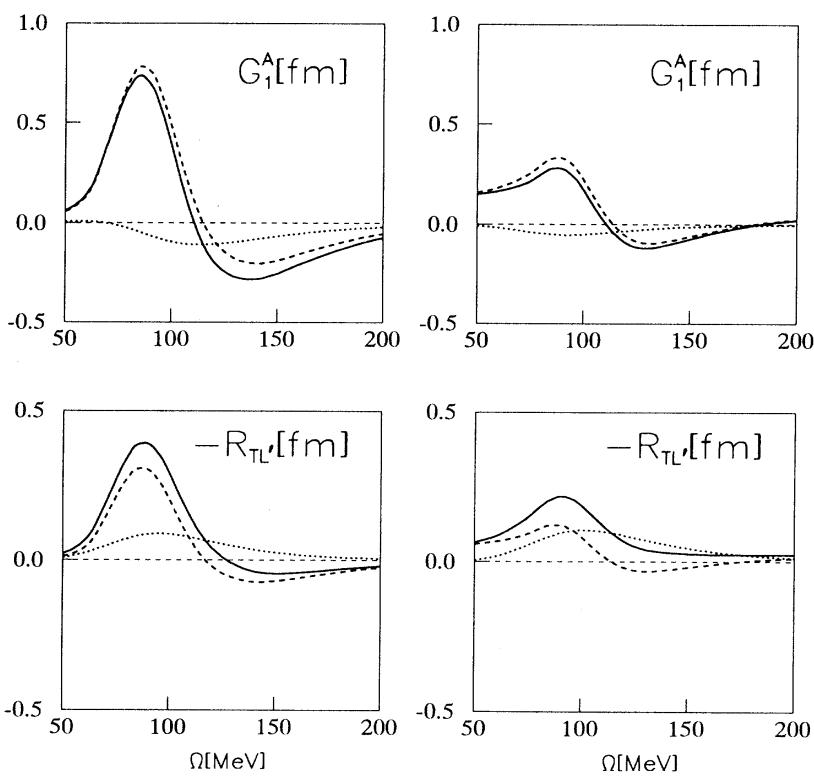

FIG. 6. ${ }^{3} \mathrm{He}$ spin structure function $G_{1}^{A}$ and the spindependent response $R_{T L^{\prime}}$ as a function of energy loss $\Omega=Q^{0}$ for a four-momentum transfer $Q^{2}=-0.16 \mathrm{GeV}^{2}$. Compared are results based on the full spectral function using extraction scheme (A) (left side) and closure using extraction scheme (C) (right side). In each case the full result (solid curve) is compared with the respective neutron (dotted curve) and proton (dashed curve) contributions. 
function according to Sec. III B and to Appendix C. The approximated spectral function $S_{\mathrm{BW}}\left(\mathrm{p}_{N} E t_{N}\right)$ is defined in Eq. (C11). Figure 3 proves that - in the considered kinematic regime-closure is for $G_{1}^{A}$ and $R_{T L}$, only a moderately accurate approximation. Figure 4 traces the deficiency of closure back to the proton contribution to $G_{1}^{A}$ and $R_{T L}$, since only for the proton can the residual two-nucleon system be in bound and in scattering states, and closure does not differentiate between them. With respect to the other structure functions $W_{1}^{A}, W_{2}^{A}$, and $G_{2}^{A}$, closure is a much better approximation. Figure 3 does not prove that point for $W_{1}^{A}$ and $W_{2}^{A}$, since it is known from Ref. [13].

(ii) In Fig. 5 results for the ${ }^{3} \mathrm{He}$ spin structure functions and spin-dependent responses are compared using the unapproximated spectral function of Sec. III B but different extraction schemes, i.e., scheme (A) of Sec. III C and schemes (B) and (C) of Appendix D. In contrast to scheme (A), scheme (B) takes all spatial current components of the nuclear current tensor form $W_{A}^{\mu \nu}\left(Q, P_{A}\right)$ in PWIA and constructs the charge components from current conservation; scheme (C) does not use the nuclear current tensor for extracting the spin structure functions, but only part of it. We are worried about scheme (C), which in our view suffers from irreparable inconsistencies as discussed in Appendix D. Thus, the large deviation between the results according to schemes (A) and (B) on one side and (C) on the other side do not reflect true theoretical error bars, but rather the shortcomings of the extraction scheme $(C)$. In contrast we take the deviation between the results based on the extraction schemes (A) and (B) as an indication for theoretical error bars. Figure 6 notes that the combination of closure approximation for the spectral function and of extraction scheme $(C)$ incorrectly overemphasizes the weight of neutron contributions to the structure function $G_{1}^{A}$ and to the spindependent response $R_{T L^{\prime}}$. That combination is used in Ref. [13].

In the result section we choose the favored extraction scheme (A) and the unapproximated spectral function of Sec. III B for the theoretical predictions.

\section{CROSS SECTION FOR SPIN-DEPENDENT INCLUSIVE ELECTRON SCATTERING}

Electron scattering from the nucleus $A$ is calculated in one-photon exchange. The fully exclusive spin-dependent cross section has the form

$$
\begin{aligned}
d \sigma\left(n_{e}, n_{A}, \beta_{X}\right)= & \frac{e_{p}^{4}}{Q^{4}} \frac{1}{4 \sqrt{\left(k_{e} \cdot P_{A}\right)^{2}-m_{e}^{2} m_{A}^{2}}}\left\langle n_{e}\left|\eta_{\mu \nu}\left(Q, k_{e}\right)\right| n_{e}\right\rangle \frac{d^{3} k_{e}^{\prime}}{2 k_{e}^{\prime 0}(2 \pi)^{3}} \\
& \times 2 P_{A}^{0}(2 \pi)^{3}\left\langle\mathbf{P}_{A} n_{A}\left|J_{A}^{\mu}(0)\right| \mathbf{P}_{X} \beta_{X}\right\rangle d^{3} P_{X}(2 \pi)^{4} \delta^{4}\left(P_{X}-Q-P_{A}\right)\left\langle\mathbf{P}_{X} \beta_{X}\left|J_{A}^{v}(0)\right| \mathbf{P}_{A} n_{A}\right\rangle
\end{aligned}
$$

The covariant form of the cross section is not manifest in Eq. (4.1). The final-state phase space

$$
(2 \pi)^{4} \delta\left(P_{X}-Q-P_{A}\right) \frac{d^{3} k_{e}^{\prime}}{2 k_{e}^{\prime 0}(2 \pi)^{3}} \frac{d^{3} P_{X}}{2 P_{X}^{0}(2 \pi)^{3}}
$$

is split up and partly recombined with the matrix elements of the nuclear current. The initial and final nuclear states, i.e., $\left|\mathbf{P}_{A} n_{A}\right\rangle$ and $\left|\mathbf{P}_{X} \beta_{X}\right\rangle$, are normalized to $\delta$ functions; this is the reason for the unusual factors in Eq. (4.1), which are absent in a relativistic description of states. The dimensionality of the differential cross section $d^{3+X} \sigma\left(n_{e}, n_{A}, \beta_{X}\right)$ depends on the amount of continuous quantum numbers needed for the description of the hadronic final state and cannot be made explicit in the general case. The electron is relativistic, i.e., $k_{e}^{0}=\left|\mathbf{k}_{e}\right|$; it undergoes the momentum transfer $Q=k_{e}-k_{e}^{\prime}$. The electron current tensor $\left\langle n_{e}\left|\eta_{\mu \nu}\left(Q, k_{e}\right)\right| n_{e}\right\rangle$ with initial polarization $n_{e}$ is

$$
\begin{aligned}
\left\langle n_{e}\left|\eta^{\mu v}\left(Q, k_{e}\right)\right| n_{e}\right\rangle & =\sum_{s_{e}^{\prime}} \bar{u}\left(\mathbf{k}_{e} n_{e}\right) \gamma^{\mu} u\left(\mathbf{k}_{e}^{\prime} s_{e}^{\prime}\right) \bar{u}\left(\mathbf{k}_{e}^{\prime} s_{e}^{\prime}\right) \gamma^{v} u\left(\mathbf{k}_{e} n_{e}\right) \\
& =2\left[k_{e}^{\prime \mu} k_{e}^{v}+k_{e}^{\mu} k_{e}^{\prime v}-g^{\mu v}\left(k_{e} \cdot k_{e}^{\prime}\right)+i m_{e} \epsilon^{\mu v \alpha \beta} Q_{\alpha} n_{e \beta}\right] .
\end{aligned}
$$

When the relativistic electron is longitudinally polarized before scattering, the polarization vector $n_{e}$ is given by

$$
n_{e}=h_{e} \frac{k_{e}}{m_{e}} \text {. }
$$

In Eq. (4.1) $4 \sqrt{\left(k_{e} \cdot P_{a}\right)^{2}-m_{e}^{2} m_{A}^{2}}$ is the flux factor in the initial state.

For the inclusive process the nuclear final state is unobserved and the cross section (4.1) is summed up with respect to the final c.m. momentum $\mathbf{P}_{X}$ and with respect to the specification $\beta_{X}$ of internal excitation. Thus, the nuclear current matrix elements can be combined into the tensor $W_{A}^{\mu \nu}\left(Q, P_{A}\right)$ of Eq. (2.1):

$$
d \sigma\left(n_{e}, n_{A}\right)=\frac{e_{p}^{4}}{Q^{4}} \frac{1}{4 \sqrt{\left(k_{e} \cdot P_{A}\right)^{2}-m_{e}^{2} m_{A}^{2}}}\left\langle n_{e}\left|\eta_{\mu \nu}\left(Q, k_{e}\right)\right| n_{e}\right\rangle 2 m_{A}(2 \pi)\left\langle n_{A}\left|W_{A}^{\mu v}\left(Q, P_{A}\right)\right| n_{A}\right\rangle \frac{d^{3} k_{e}^{\prime}}{2 k_{e}^{\prime 0}(2 \pi)^{3}} .
$$

The cross section is calculated in the laboratory system, i.e., in the nuclear c.m. system with $\mathbf{P}_{A}=0$. The electron un- 
dergoes the energy loss $\Omega=Q^{0}=\left(Q \cdot P_{A}\right) / m_{A}$; its four-momentum in the final state is $k_{e}^{\prime}=\left(\left|\mathbf{k}_{e}^{\prime}\right|,\left|\mathbf{k}_{e}^{\prime}\right| \Omega_{e}^{\prime}\right)$. The scattering angle of the electron is $\Theta_{e} ; \sigma_{\mathrm{Mott}}=\left(e_{p}^{2} / 8 \pi k_{e}^{0}\right)^{2} \cos ^{2} \frac{1}{2} \Theta_{e} / \sin ^{4} \frac{1}{2} \Theta_{e}$ is the Mott cross section. The target polarization $n_{A}=\left(0, \hat{\mathbf{n}}_{A}\right)$ is parametrized by either of the two sets of angles $\left(\theta_{A}, \phi_{A}\right)$ or $\left(\theta^{*}, \phi^{*}\right)$ :

$$
\begin{aligned}
& \hat{\mathbf{n}}_{A}=\widehat{\mathbf{e}}_{S} \cos \phi_{A} \sin \theta_{A}+\widehat{\mathbf{e}}_{N} \sin \phi_{A} \sin \theta_{A}+\widehat{\mathbf{e}}_{L} \cos \theta_{A}, \\
& \hat{\mathbf{n}}_{A}=\widehat{\mathbf{e}}_{x} \cos \phi^{*} \sin \theta^{*}+\widehat{\mathbf{e}}_{y} \sin \phi^{*} \sin \theta^{*}+\widehat{\mathbf{e}}_{z} \cos \theta^{*},
\end{aligned}
$$

with the unit vectors $\widehat{\mathbf{e}}_{L}=\widehat{\mathbf{k}}_{e}, \widehat{\mathbf{e}}_{N}=\widehat{\mathbf{k}}_{e} \times \widehat{\mathbf{k}}_{e}^{\prime} /\left|\widehat{\mathbf{k}}_{e} \times \widehat{\mathbf{k}}_{e}^{\prime}\right|$, and $\widehat{\mathbf{e}}_{S}=\widehat{\mathbf{e}}_{N} \times \widehat{\mathbf{e}}_{L}$ and the unit vectors $\widehat{\mathbf{e}}_{z}=\widehat{\mathbf{Q}}$, $\widehat{\mathbf{e}}_{y}=\widehat{\mathbf{e}}_{N}$, and $\widehat{\mathbf{e}}_{x}=\hat{\mathbf{e}}_{y} \times \widehat{\mathbf{e}}_{z}$ according to Fig. 7. We note that for fixed angles $\left(\theta_{A}, \phi_{A}\right)$ the angles $\left(\theta^{*}, \phi^{*}\right)$ are not fixed, but depend on the momentum transfer $\mathbf{Q}$. With the unit vectors $\widehat{\mathbf{e}}_{1}, \widehat{\mathbf{e}}_{2}$, and $\widehat{\mathbf{e}}_{3}$ of Sec. II given entirely by nuclear quantities, the form (2.4b) for the nuclear polarization vector $n_{A}$ always results, e.g., $n_{A}=\left(0, \sin \theta^{*}, 0, \cos \theta^{*}\right)$ for the polarization. angles $\left(\theta^{*}, \phi^{*}\right)$, the angle $\phi^{*}$ being absorbed in the definition of the unit vectors. Once the tensor contractions are carried out in Eq. (4.3), the spin-dependent differential cross section with longitudinal electron polarization is obtained in the nuclear c.m. system:

$$
\begin{aligned}
\frac{d^{2} \sigma}{d k_{e}^{\prime 0} d \Omega_{e}^{\prime}}\left(h_{e}, \hat{\mathbf{n}}_{A}\right)=\sigma_{\text {Mott }}[ & W_{2}^{A}\left(Q^{2}, \Omega\right)+2 \tan ^{2} \frac{1}{2} \Theta_{e} W_{1}^{A}\left(Q^{2}, \Omega\right) \\
& +2 h_{e} \tan ^{2} \frac{1}{2} \Theta_{e}\left[\frac{G_{1}^{A}\left(Q^{2}, \Omega\right)}{m_{A}}\left[k_{e}^{0} \cos \theta_{A}+k_{e}^{\prime 0}\left(\cos \Theta_{e} \cos \theta_{A}+\sin \Theta_{e} \sin \theta_{A} \cos \phi_{A}\right)\right]\right. \\
& \left.-2 \frac{G_{2}^{A}\left(Q^{2}, \Omega\right)}{m_{A}^{2}} k_{e}^{0} k_{e}^{\prime 0}\left[\cos \theta_{A}-\left(\cos \Theta_{e} \cos \theta_{A}+\sin \Theta_{e} \sin \theta_{A} \cos \phi_{A}\right)\right]\right],
\end{aligned}
$$

or, equivalently [12],

$$
\begin{aligned}
\frac{d^{2} \sigma}{d k_{e}^{\prime 0} d \Omega_{e}^{\prime}}\left(h_{e}, \hat{\mathbf{n}}_{A}\right)=\sigma_{\mathrm{Mott}} & \frac{Q^{4}}{\mathbf{Q}^{4}} R_{L}\left(Q^{2}, \Omega\right)+\left[-\frac{1}{2} \frac{Q^{2}}{\mathbf{Q}^{2}}+\tan ^{2} \frac{1}{2} \Theta_{e}\right] R_{T}\left(Q^{2}, \Omega\right) \\
& \left.+h_{e} \tan \frac{1}{2} \Theta_{e}\left[\left[-\frac{Q^{2}}{\mathbf{Q}^{2}}+\tan ^{2} \frac{1}{2} \Theta_{e}\right]^{1 / 2} R_{T^{\prime}}\left(Q^{2}, \Omega\right) \cos \theta^{*}+\frac{1}{\sqrt{2}} \frac{Q^{2}}{Q^{2}} R_{T L^{\prime}}\left(Q^{2}, \Omega\right) \sin \theta^{*} \cos \phi^{*}\right]\right\}
\end{aligned}
$$

In experiments the asymmetry $A\left(k_{e}^{\prime 0}, \Omega_{e}^{\prime}, \hat{\mathbf{n}}_{A}\right)$ is observed. It is defined by

$$
A\left(k_{e}^{\prime 0}, \Omega_{e}^{\prime}, \widehat{\mathbf{n}}_{A}\right)=\frac{\frac{d^{2} \sigma}{d k_{e}^{\prime 0} d \Omega_{e}^{\prime}}\left(+1, \widehat{\mathbf{n}}_{A}\right)-\frac{d^{2} \sigma}{d k_{e}^{\prime 0} d \Omega_{e}^{\prime}}\left(-1, \widehat{\mathbf{n}}_{A}\right)}{\frac{d^{2} \sigma}{d k_{e}^{\prime 0} d \Omega_{e}^{\prime}}\left(+1, \hat{\mathbf{n}}_{A}\right)+\frac{d^{2} \sigma}{d k_{e}^{\prime 0} d \Omega_{e}^{\prime}}\left(-1, \widehat{\mathbf{n}}_{A}\right)} .
$$

It takes the explicit form

$$
\begin{aligned}
A\left(k_{e}^{\prime 0}, \Omega_{e}^{\prime}, \hat{\mathbf{n}}_{A}\right)= & 2 \tan ^{2} \frac{1}{2} \Theta_{e}\left[\frac{G_{1}^{A}\left(Q^{2}, \Omega\right)}{m_{A}}\left[k_{e}^{0} \cos \theta_{A}+k_{e}^{\prime 0}\left(\cos \Theta_{e} \cos \theta_{A}+\sin \Theta_{e} \sin \theta_{A} \cos \phi_{A}\right)\right]\right. \\
& \left.-2 \frac{G_{2}^{A}\left(Q^{2}, \Omega\right)}{m_{A}^{2}} k_{e}^{0} k_{e}^{\prime 0}\left[\cos \theta_{A}-\left(\cos \Theta_{e} \cos \theta_{A}+\sin \Theta_{e} \sin \theta_{A} \cos \phi_{A}\right)\right]\right) \\
& \times\left[W_{2}^{A}\left(Q^{2}, \Omega\right)+2 \tan ^{2} \frac{1}{2} \Theta_{e} W_{1}^{A}\left(Q^{2}, \Omega\right)\right]^{-1}
\end{aligned}
$$

or equivalently [12], 


$$
\begin{aligned}
A\left(k_{e}^{\prime 0}, \Omega_{e}^{\prime}, \hat{\mathbf{n}}_{A}\right)= & \left\{\tan \frac{1}{2} \Theta_{e}\left[\left[-\frac{Q^{2}}{\mathbf{Q}^{2}}+\tan ^{2} \frac{1}{2} \Theta_{e}\right]^{1 / 2} R_{T^{\prime}}\left(Q^{2}, \Omega\right) \cos \theta^{*}+\frac{1}{\sqrt{2}} \frac{Q^{2}}{Q^{2}} R_{T L^{\prime}}\left(Q^{2}, \Omega\right) \sin \theta^{*} \cos \phi^{*}\right]\right\} \\
& \times\left[\frac{Q^{4}}{\mathbf{Q}^{4}} R_{L}\left(Q^{2}, \Omega\right)+\left[-\frac{1}{2} \frac{Q^{2}}{\mathbf{Q}^{2}}+\tan ^{2} \frac{1}{2} \Theta_{e}\right] R_{T}\left(Q^{2}, \Omega\right)\right]^{-1} .
\end{aligned}
$$

Using the notation $\widehat{\mathbf{n}}_{A}=\widehat{\mathbf{n}}_{A}\left(\theta_{A}, \phi_{A}\right)=\widehat{\mathbf{n}}_{A}\left(\theta^{*}, \phi^{*}\right)$ we note the symmetry relations

$$
\begin{aligned}
& A\left(k_{e}^{\prime 0}, \Omega_{e}^{\prime}, \hat{\mathbf{n}}_{A}\left(\theta_{A}, \phi_{A}\right)\right)=A\left(k_{e}^{\prime 0}, \Omega_{e}^{\prime}, \widehat{\mathbf{n}}_{A}\left(-\theta_{A}, \phi_{A}+\pi\right)\right)=-A\left(k_{e}^{\prime 0}, \Omega_{e}^{\prime}, \hat{\mathbf{n}}_{A}\left(\theta_{A}+\pi, \phi_{A}\right)\right), \\
& A\left(k_{e}^{\prime 0}, \Omega_{e}^{\prime}, \hat{\mathbf{n}}_{A}\left(\theta^{*}, \phi^{*}\right)\right)=A\left(k_{e}^{\prime 0}, \Omega_{e}^{\prime}, \widehat{\mathbf{n}}_{A}\left(-\theta^{*}, \phi^{*}+\pi\right)\right)=-A\left(k_{e}^{\prime 0}, \Omega_{e}^{\prime}, \widehat{\mathbf{n}}_{A}\left(\theta^{*}+\pi, \phi^{*}\right)\right),
\end{aligned}
$$

which are employed for combining experimental data. For later purposes the spin-dependent cross section (4.5) and the asymmetry (4.7) are given in the two different parametrizations of the nuclear polarization vector $\hat{\mathbf{n}}_{A}$ according to Eq. (4.4), once combined with the nuclear structure functions, once with the nuclear responses.
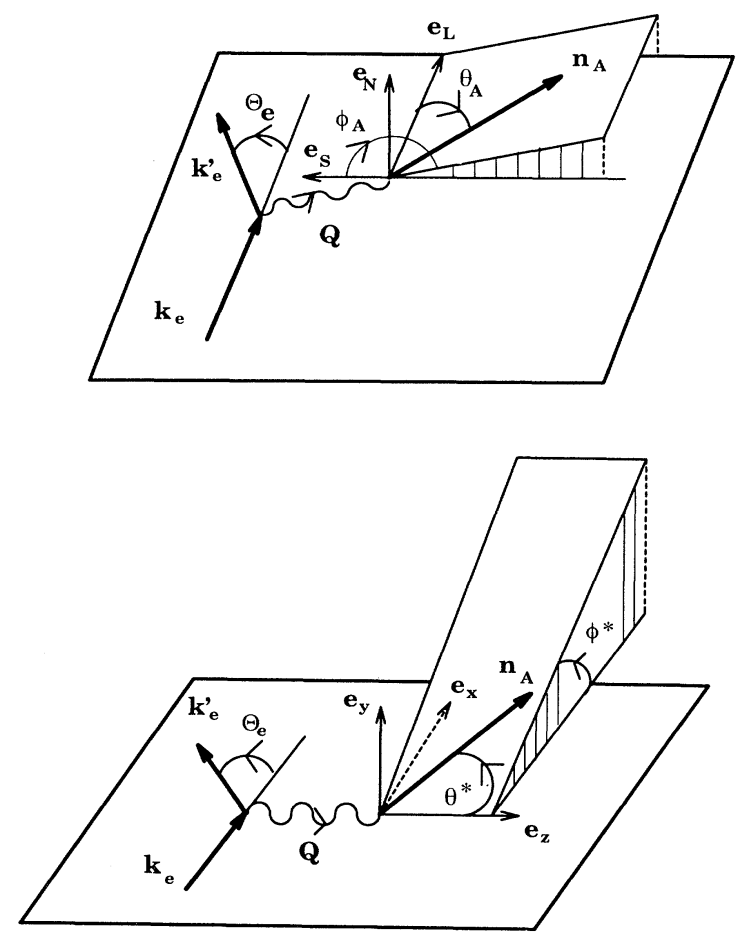

FIG. 7. Illustration of two different parametrizations for the target polarization vector $\hat{\mathbf{n}}_{A}$ according to Eq. (4.5). The scattering plane is defined by the momenta $\mathbf{k}_{e}$ and $\mathbf{k}_{e}^{\prime}$ of the incoming and outgoing electrons, respectively. On the top, the parametrization is in terms of the angles $\left(\theta_{A}, \phi_{A}\right)$; the shaded area lies in the plane defined by the unit vectors $\widehat{\mathbf{e}}_{S}$ and $\hat{\mathbf{e}}_{N}$; the projection of $\hat{\mathbf{n}}_{A}$ onto that plane forms the angle $\phi_{A}$ with the direction $\hat{\mathbf{e}}_{S}$. On the bottom, the parametrization is in terms of the angles $\left(\theta^{*}, \phi^{*}\right)$; the shaded area lies in the $x-y$ plane; the projection of $\widehat{\mathbf{n}}_{A}$ onto that plane forms the angle $\phi^{*}$ with the $x$ direction; the true unit vector $\widehat{\mathbf{e}}_{x}$ originating from the origin as $\hat{\mathbf{e}}_{y}$ and $\hat{\mathbf{e}}_{z}$ is hidden in the figure and is therefore only indicated by a dashed arrow. When the target polarization lies in the scattering plane, the angles $\phi_{A}$ and $\phi^{*}$ are both zero or $\pi$.

\section{RESULTS}

All theoretical predictions of this paper are based on the spectral function derived from the Paris potential [14]. The trinucleon bound-state wave function $\left|\mathbf{P}_{A} s_{A}\right\rangle$ is taken from Ref. [15], which updates the technique of Ref. [16]; the wave function is computed from momentum-space Faddeev equations with 18 distinct Faddeev channels and expanded into 22 partial-wave components according to Fig. 1 of Ref. [16]; the mesh is specialized for the calculation of this paper. The predictions refer to the kinematic regime of quasielastic scattering. Throughout this paper the elastic proton $\left[p=N\left(\frac{1}{2}\right)\right]$ and neutron $\left[n=N\left(-\frac{1}{2}\right)\right]$ structure functions are used according to Eqs. (3.3) in the form factor parametrization of Ref. [17], i.e.,

$$
G_{E}^{n}\left(Q_{N}^{2}\right)=\mu_{n} \tau(1+5.6 \tau)^{-1} G_{E}^{P}\left(Q_{N}^{2}\right),
$$

with the dipole dependence

$$
\begin{aligned}
G_{E}^{P}\left(Q_{N}^{2}\right)=G_{M}^{P}\left(Q_{N}^{2}\right) / \mu_{p} & =G_{M}^{n}\left(Q_{N}^{2}\right) / \mu_{n} \\
& =\left[1-Q_{N}^{2} /\left(0.71 \mathrm{GeV}^{2}\right)\right]^{-2}
\end{aligned}
$$

In Eq. (5.1) $\tau=-Q_{N}^{2} / 4 m_{N}^{2}$ and $\mu_{p}$ and $\mu_{n}$ are the proton and neutron magnetic moments in units of the nuclear magneton. That simple form factor parametrization is chosen in order to allow a convenient comparison with results of Ref. [13]. Using an improved form factor parametrization instead of Eq. (5.1b) changes the theoretical results presented in Figs. 9 and 10 by less than $6 \%$.

Theoretical results for the asymmetry in spindependent quasielastic scattering of longitudinally polarized electrons from a polarized ${ }^{3} \mathrm{He}$ target are calculated. They refer to measurements under way at MIT/Bates. First results are given in Refs. [18-21]. The measurements use longitudinally polarized electrons of energy $k_{e}^{0}=574 \mathrm{MeV}$. In both experiments the target polarization is confined to the scattering plane and kept fixed in the experiments, i.e., $\phi_{A}=0$ or $\pi$. Even when the polarization angle $\theta_{A}$ is fixed, the angle $\theta^{*}$ varies with energy loss for both experiments, since in the experiments energy loss and momentum transfer $\mathbf{Q}$ are related.

In the experiment of Ref. [18] the electrons are scattered under the angle $\Theta_{e}=44^{\circ}$ for different values of the energy loss $\Omega$. Thus, the data do not refer to a fixed 

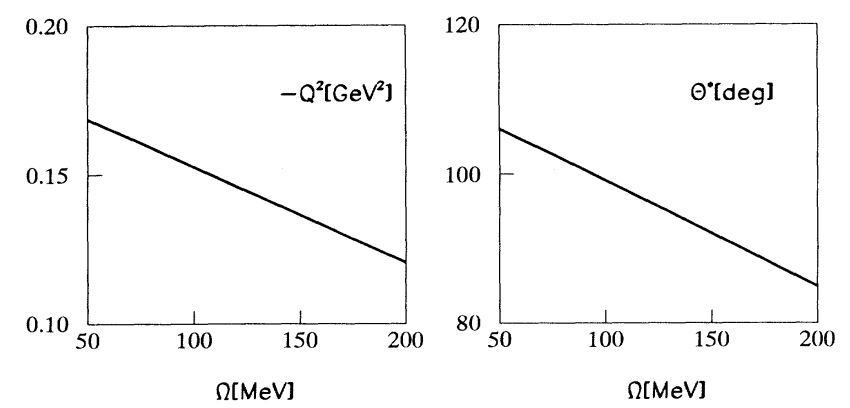

FIG. 8. Four-momentum transfer $Q^{2}$ as a function of energy loss $\Omega=Q^{0}$ for electrons with an initial energy $k_{e}^{0}=574 \mathrm{MeV}$ and with a scattering angle $\Theta_{e}=44^{\circ}$ on the left side, polarization angle $\theta^{*}$ of Fig. 7 as a function of energy loss $\Omega=Q^{0}$ for electrons with the same initial energy $k_{e}^{0}=574 \mathrm{MeV}$, with the same scattering angle $\Theta_{e}=44^{\circ}$ and with a target polarization $\left(\theta_{A}, \phi_{A}\right)=\left(44.5^{\circ}, 0\right)$ confined to the scattering plane on the right side.

four-momentum transfer $Q^{2}$, though its variation over the measured energy loss is rather small. Figure 8 illustrates the small variation of $Q^{2}$ for the experiment of Ref. [18]; its value in the quasielastic peak is $Q^{2}=-0.16$ $\mathrm{GeV}^{2}$, the value used in Sec. III for examples of the ${ }^{3} \mathrm{He}$ structure functions and responses. Figure 8 also indicates the variation of the polarization angle $\theta^{*}$. At $\theta^{*}=\pi / 2$ the experiment would see the mixed transverselongitudinal response $R_{T L^{\prime}}$ exclusively. That situation is never reached. The theoretical prediction for that experiment together with the existing experimental data are given in Fig. 9 and Table I.

In the experiment of Refs. [19-21] the electrons are scattered under the angle $\Theta_{e}=51.1^{\circ}$. In principle they could be observed as a function of energy loss $\Omega$, but only the asymmetry averaged over energy loss or at a mean value close to the quasielastic peak is measured. At the polarization angle $\theta^{*}=0$ the experiment would see the transverse response $R_{T^{\prime}}$ exclusively. That situation is almost reached. We are puzzled by the fact that the data

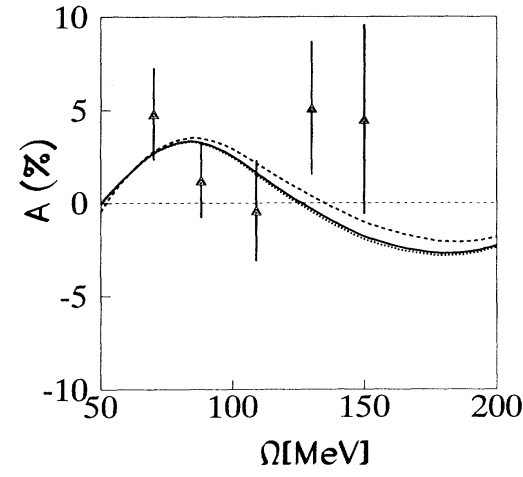

FIG. 9. Asymmetry in inclusive electron scattering from polarized ${ }^{3} \mathrm{He}$ as a function of the energy loss $\Omega=Q^{0}$. The experimental and theoretical results refer to the initial electron energy $k_{e}^{0}=574 \mathrm{MeV}$ and the scattering angle $\Theta_{e}=44^{\circ}$. The experimental data are from Ref. [18]; they are derived from two physically distinct target polarizations according to Table I; a weighted average is formed with respect to charge hours. Theoretical results are given for the weighted average in the solid curve, for the pure target polarization $\left(\theta_{A}, \phi_{A}\right)=\left(51.5^{\circ}, 0\right)$ in the dashed curve and for the pure target polarization $\left(\theta_{A}, \phi_{A}\right)=\left(44.5^{\circ}, 0\right)$ in the dotted curve.

of Refs. [19,20] and of Ref. [21] disagree substantially. Reference [21] also gives one data point for a polarization angle $\theta^{*}=\pi / 2$ corresponding to the mixed transverselongitudinal response $R_{T L^{\prime}}$ exclusively. All data are collected in Table II. The theoretical prediction for those experiments together with the existing data points are given in Fig. 10 and Table II.

The spin of ${ }^{3} \mathrm{He}$ is carried to a large extent by the neutron. The effective neutron polarization

$P_{n}=\left\langle\mathbf{P}_{A} s_{A}=\frac{1}{2}\left|\sum_{i} \frac{1}{2}\left[1-\tau_{z}(i)\right] \sigma_{z}(i)\right| \mathbf{P}_{A} s_{A}=\frac{1}{2}\right\rangle$

in the polarized ${ }^{3} \mathrm{He}$ is $P_{n}=88.1 \%$ for the trinucleon wave function employed in the calculations of this paper. That value is quite characteristic for wave functions de-

TABLE I. Asymmetry measurement of Ref. [18]. The data refer to the measurement of Ref. [18] with the beam energy $k_{e}^{0}=574 \mathrm{MeV}$ and the electron scattering angle of $\Theta_{e}=44.0^{\circ}$. Column 1 gives the charge hours during which data were collected, and columns 2-5 give the polarization angles of the polarized ${ }^{3} \mathrm{He}$ target, the symmetry relation of Eq. (4.8) being used in order to combine the data in rows 2 and 3. The quoted experimental asymmetry $A_{\text {exp }}$ combines data for an energy loss $57 \leq \Omega \leq 160 \mathrm{MeV}$ with the position of the quasielastic peak at $\Omega_{\text {peak }}=86 \mathrm{MeV}$. The quoted theoretical asymmetry $A_{\text {theo }}$ is the corresponding average over the energy loss $57 \leq \Omega \leq 160 \mathrm{MeV}$. The last row contains the average of the experimental and theoretical asymmetry over the data weighted by charge hours for the two distinct polarizations; the first uncertainty is statistical as in column 6 , the second is systematic. Figure 9 presents data in the same weighted average, though as function of energy loss $\Omega$.

\begin{tabular}{ccccccr}
\hline \hline $\begin{array}{l}\text { Charge } \\
(\mu \boldsymbol{A} \text { h) }\end{array}$ & $\begin{array}{c}\theta_{A} \\
(\mathrm{deg})\end{array}$ & $\begin{array}{c}\phi_{A} \\
(\mathrm{deg})\end{array}$ & $\begin{array}{c}\theta^{*}\left(\Omega_{\text {peak }}\right) \\
(\mathrm{deg})\end{array}$ & $\begin{array}{c}\phi^{*}\left(\Omega_{\text {peak }}\right) \\
(\mathrm{deg})\end{array}$ & $\begin{array}{c}A_{\text {exp }} \\
(\%)\end{array}$ & $\begin{array}{r}A_{\text {theo }} \\
(\%)\end{array}$ \\
\hline 228 & 51.5 & 0 & 108.4 & 0 & $3.2 \pm 2.7$ & 1.47 \\
336 & 44.5 & 0 & 101.4 & 0 & $28 \pm 2.6$ & 0.93 \\
808 & 135.5 & 180 & 78.6 & 180 & $-1.9 \pm 1.7$ & -0.93 \\
& & & & $2.38 \pm 1.27 \pm 0.44$ & 1.02 \\
\hline
\end{tabular}


TABLE II. Asymmetry measurement of Refs. [19-21]. The data refer to three distinct measurements of Refs. [19-21] with the beam energy $k_{e}^{0}=574 \mathrm{MeV}$, the electron scattering angle of $\Theta_{e}=51.1^{\circ}$, and the position of the quasielastic peak at $\Omega_{\text {peak }}=106 \mathrm{MeV}$. The lines in the table separate the entries from different measurements; the data of the first measurement are given in rows 1-4, those of the second in rows 5-7, and those of the third in row 8 . Column 1 gives the charge hours, if documented, during which data were collected, and columns 2-5 give the polarization angles of the polarized ${ }^{3} \mathrm{He}$ target, the symmetry relation of Eq. (4.8) being used in order to combine the data in rows 2 and 3 and in rows 5 and 6, respectively. Rows $1-4$ present the data of Ref. [19] in the analysis of Ref. [20]. In that case, the experimental asymmetry $A_{\text {exp }}$ combines data for an energy loss $90 \leq \Omega \leq 138 \mathrm{MeV}$. The quoted theoretical asymmetry $A_{\text {theo }}$ is the corresponding average over the same energy loss $90 \leq \Omega \leq 138$ $\mathrm{MeV}$; in parentheses $A_{\text {theo }}$ is given for the energy loss $\Omega=114 \mathrm{MeV}$ without averaging. Row 4 contains the average of the experimental and theoretical asymmetry over the data weighted by charge hours for the two distinct polarizations; the first uncertainty is statistical as in column 6 , the second is systematic. Figure 10 presents the single data point and the theoretical prediction in the same weighted average, though as function of energy loss $\Omega$. Rows 5-8 present the data of Ref. [21]. Row 7 presents data for the polarization of rows 5 and 6 combined by symmetry. The polarization comparable with those of Refs. [19,20]. In Ref. [21] a spread in energy loss $\Omega$ is not reported; the theoretical asymmetry $A_{\text {theo }}$ is only given for the mean value of energy loss $\Omega=116 \mathrm{MeV}$ without averaging and therefore listed in parentheses in correspondence with the upper entry in the table.

\begin{tabular}{|c|c|c|c|c|c|c|}
\hline $\begin{array}{l}\text { Charge } \\
(\mu A \mathrm{~h}) \\
\end{array}$ & $\begin{array}{c}\theta_{A} \\
(\mathrm{deg})\end{array}$ & $\begin{array}{c}\phi_{A} \\
(\mathrm{deg})\end{array}$ & $\begin{array}{c}\theta^{*}\left(\Omega_{\text {peak }}\right) \\
(\mathrm{deg})\end{array}$ & $\begin{array}{c}\phi^{*}\left(\Omega_{\text {peak }}\right) \\
(\mathrm{deg})\end{array}$ & $\begin{array}{l}A_{\exp } \\
(\%)\end{array}$ & $\begin{array}{l}A_{\text {theo }} \\
(\%)\end{array}$ \\
\hline 239 & 51.5 & 180 & 0.9 & 0 & $-3.8 \pm 3.0$ & $-4.7(-4.5)$ \\
\hline 333 & 44.5 & 180 & 7.9 & 0 & $-2.5 \pm 2.9$ & $-4.4(-4.1)$ \\
\hline 808 & 135.5 & 0 & 172.1 & 180 & $4.4 \pm 2.8$ & $4.4(4.1)$ \\
\hline \multicolumn{5}{|c|}{1381 (combined) } & $-3.79 \pm 1.37 \pm 0.67$ & $-4.5(-4.2)$ \\
\hline & 49.2 & 180 & 3.2 & 0 & & \\
\hline & 130.8 & 0 & 176.8 & 180 & & \\
\hline & & & & & $-2.6 \pm 0.90 \pm 0.46$ & $(-4.7)$ \\
\hline & 37.8 & 0 & 90.2 & 0 & $1.75 \pm 1.20 \pm 0.31$ & (2.9) \\
\hline
\end{tabular}

rived from most realistic interactions [22] and therefore may be taken as a fact of nature. Thus, the experimenters of Refs. [18-21] hope that the polarized ${ }^{3} \mathrm{He}$ target can effectively be taken as a neutron spin target and therefore hope to explore electromagnetic properties of the neutron. The asymmetry experiments measure the ${ }^{3} \mathrm{He}$ spin structure functions and spin-dependent responses, to which the neutron and the two protons make distinctive contributions in PWIA. Figure 11 displays the ${ }^{3} \mathrm{He}$ spin structure functions $G_{1}^{A}$ and $G_{2}^{A}$ as well as the corresponding spin-dependent responses $R_{T L^{\prime}}$ and $R_{T^{\prime}}$ and splits them up according to the contributions of individual nucleons. The structure functions and responses are plotted as a function of energy loss for the experimental kinematics $k_{e}^{0}=574 \mathrm{MeV}$ and $\Theta_{e}=44^{\circ}$ of Ref. [18], i.e., the four-momentum transfer $Q^{2}$ is not fixed but varies according to Fig. 8 . The neutron dominates the response $R_{T^{\prime}}$ and the structure function $G_{2}^{A}$. But those functions are derived from the neutron's spatial current and are rather well known in their important spin parts. Thus, the neutron-dominated response $R_{T^{\prime}}$ and spin structure function $G_{2}^{A}$ do not yield novel neutron properties. In contrast, the response $R_{T L^{\prime}}$ and the spin structure function $G_{1}^{A}$ depend on the rather unknown neutron charge property. However, that neutron contribution to $R_{T L^{\prime}}$ and $G_{1}^{A}$ is too small to make its accurate determination easy; in fact, that neutron contribution is overwhelmed by the corresponding proton contribution.

In this context we recall that according to Sec. III D the closure approximation in the spectral function and the adoption of the extraction scheme (C) of Appendix D work poorest for the spin structure function $G_{1}^{A}$ and the spin-dependent response $R_{T L^{\prime}}$. The combined use of both erroneously emphasizes the neutron contribution to $G_{1}^{A}$

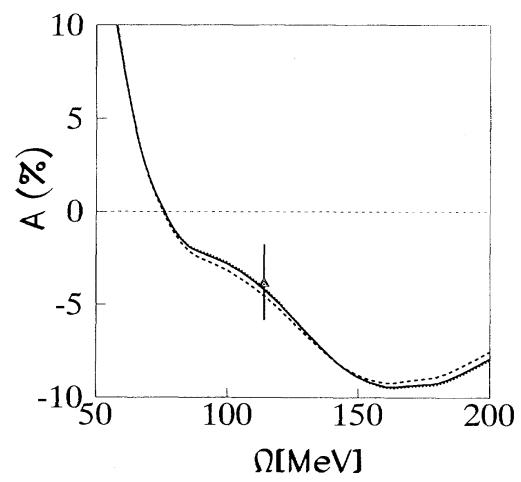

FIG. 10. Asymmetry in inclusive electron scattering from polarized ${ }^{3} \mathrm{He}$ as a function of the energy loss $\Omega=Q^{0}$. The experimental and theoretical results refer to the initial electron energy $k_{e}^{0}=574 \mathrm{MeV}$ and the scattering angle $\Theta_{e}=51.1^{\circ}$. The experimental data point is from Refs. $[19,20]$; it is derived from two physically distinct target polarizations according to Table 2; a weighted average is formed with respect to charge hours. Theoretical results are given for the weighted average in the solid curve, for the pure target polarization $\left(\theta_{A}, \phi_{A}\right)=\left(51.5^{\circ}, 180^{\circ}\right)$ in the dashed curve and for the pure target polarization $\left(\theta_{A}, \phi_{A}\right)=\left(44.5^{\circ}, 180^{\circ}\right)$ in the dotted curve. 

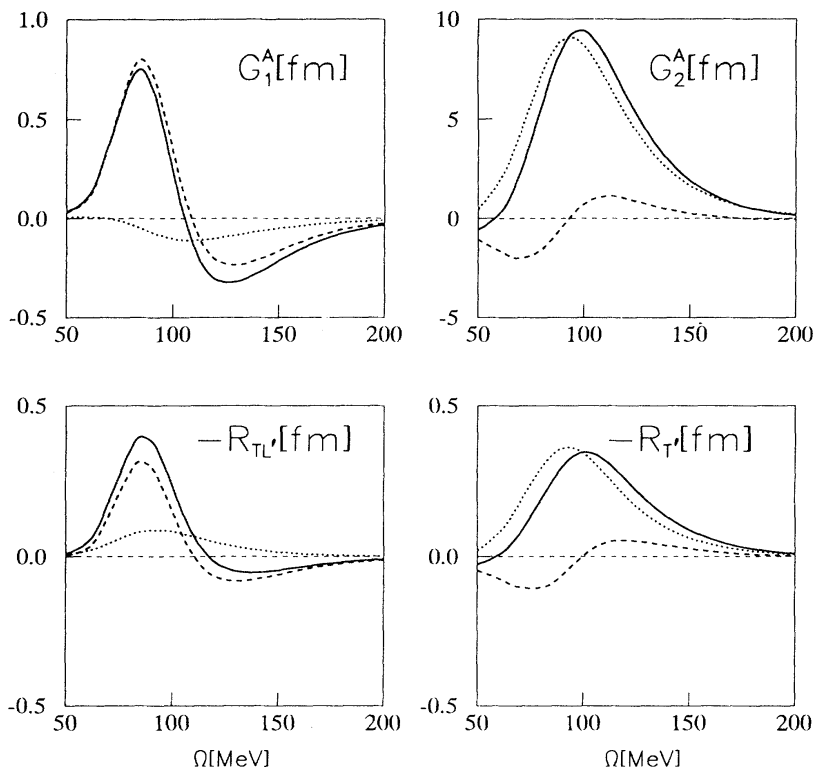

FIG. 11. ${ }^{3} \mathrm{He}$ spin structure functions $G_{1}^{A}$ and $G_{2}^{A}$ and spindependent responses $R_{T L}$, and $R_{T^{\prime}}$ as a function of energy loss $\Omega=Q^{0}$. The theoretical results refer to the initial electron energy $k_{e}^{0}=574 \mathrm{MeV}$ and the scattering angle $\Theta_{e}=44^{\circ}$. Thus, the four-momentum transfer $Q^{2}$ varies in the plotted function with energy loss $\Omega=Q^{0}$ according to Fig. 8. The full result (solid curve) is compared with the respective neutron (dotted curve) and proton (dashed curve) contributions.

and $R_{T L^{\prime}}$ as Fig. 6 illustrates. Reference [13] makes use of a spectral function in closure approximation and uses extraction scheme (C). The analysis of the two asymmetry experiments has been based on the results of Ref. [13]. We admit to being puzzled by the fact that the predictions of this paper, though believed to be theoretically

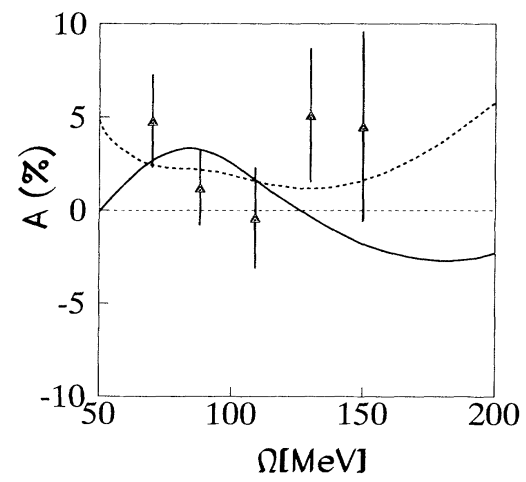

FIG. 12. Asymmetry in inclusive electron scattering from polarized ${ }^{3} \mathrm{He}$ as a function of the energy loss $\Omega=Q^{0}$. The experimental and theoretical results refer to the initial electron energy $k_{e}^{0}=574 \mathrm{MeV}$ and the scattering angle $\theta_{e}=44^{\circ}$. The experimental data are from Ref. [18] as in Fig. 9. Two theoretical predictions are compared, i.e., the prediction, based on the full spectral function of Eq. (3.4) and on the favored extraction scheme (A) of Eq. (3.11) and (D2), shown as solid curve, is compared with the prediction, based on the closure approximation for the spectral function according to Eq. (C11) and on the questionable extraction scheme (C) of Eq. (D6), shown as dashed curve. better founded, are in much poorer agreement with the experimental data than predictions using the calculational scheme of Ref. [13]. That fact is shown in Fig. 12.

\section{CONCLUSION}

This paper studies spin-dependent inelastic electron scattering from polarized ${ }^{3} \mathrm{He}$. The theoretical description uses the plane-wave impulse approximation. The final-state interaction between the knocked-out nucleon or the inelastically produced hadrons and the residual spectator nucleons as well as meson-exchange contributions to the nuclear current are neglected. The given theoretical description applies to inclusive processes in all kinematic regimes of inelastic electron scattering. Calculational examples are given for the ${ }^{3} \mathrm{He}$ structure functions and responses in the region of the quasielastic scattering. In that kinematic regime a detailed comparison of theoretical predictions and existing experimental data for the asymmetry is carried out.

The polarized ${ }^{3} \mathrm{He}$ target is indeed a good neutron spin target, though we are pessimistic with respect to extracting neutron charge properties from inclusive quasielastic electron scattering. The results illustrated in Fig. 11 lead to that pessimism. In the past, the authors of this paper often called for corresponding experiments with polarized ${ }^{3} \mathrm{H}$ as conclusive tests. Polarized ${ }^{3} \mathrm{H}$ is as effective a proton spin target as ${ }^{3} \mathrm{He}$ is a neutron target, and we believed that, once ${ }^{3} \mathrm{H}$ experiments could yield the wellknown electromagnetic proton properties, it would be proven that the ${ }^{3} \mathrm{He}$ experiments would be successful in
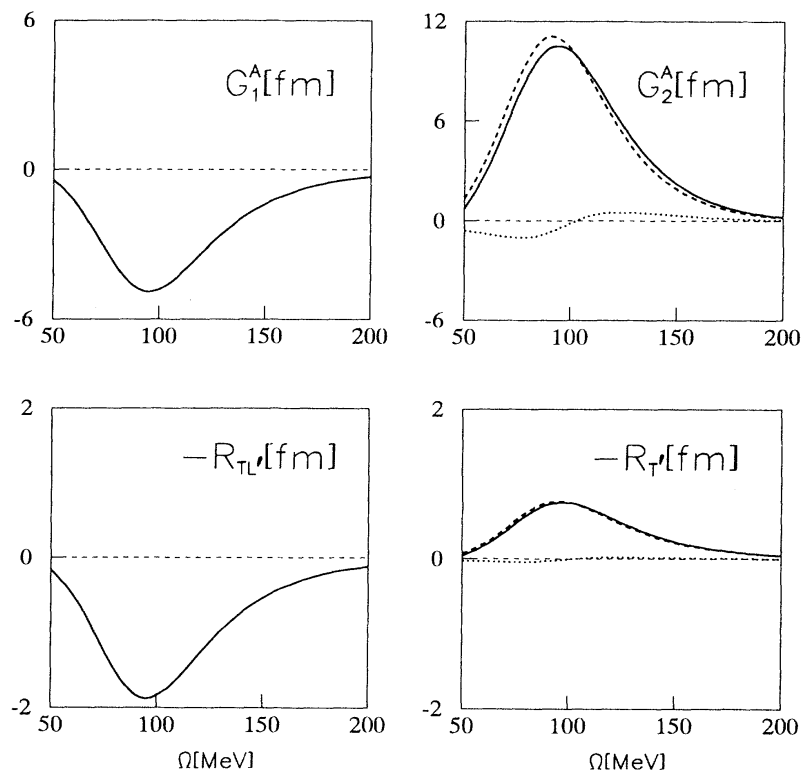

FIG. 13. ${ }^{3} \mathrm{H}$ spin structure functions $G_{1}^{A}$ and $G_{2}^{j A}$ and spindependent responses $R_{T L^{\prime}}$ and $R_{T^{\prime}}$ as a function of energy loss $\Omega=Q^{0}$ for a four-momentum transfer $Q^{2}=-0.16 \mathrm{GeV}^{2}$. The full result (solid curve) is compared with the respective neutron (dotted curve) and proton (dashed curve) contributions. For $G_{1}^{A}$ and $R_{T L^{\prime}}$ the proton contribution coincides with the full result and the neutron contribution coincides with the zero line; they are therefore not indicated for $G_{1}^{A}$ and $R_{T L^{\prime}}$. 
providing the unknown charge neutron properties. We now consider that misleading reasoning, since there is a subtle difference between the two targets: At low momentum transfer the proton charge form factor is large, whereas the neutron one is comparatively small. Indeed, as Fig. 13 demonstrates, the ${ }^{3} \mathrm{H}$ response $R_{T L^{\prime}}$ is almost exclusively given by the proton; the measurement of $R_{T L}$, in ${ }^{3} \mathrm{H}$ can therefore determine the proton charge properties. In contrast, the ${ }^{3} \mathrm{He}$ response $R_{T L^{\prime}}$ is given by the neutron only to a minor degree. Thus, a successful ${ }^{3} \mathbf{H}$ experiment for proton properties does not at all prove the feasibility of extracting neutron properties from the corresponding ${ }^{3} \mathrm{He}$ experiments.

The developed theoretical apparatus is also applicable to inelastic lepton scattering in the kinematic regime of quasifree pion production and in the deep-inelastic regime. In those regimes the proton and neutron structure functions become comparable. Indeed, in those regimes [23] a polarized ${ }^{3} \mathrm{He}$ target may be employed to extract spin structure functions of the neutron successfully from ${ }^{3} \mathrm{He}$ measurements.

\section{ACKNOWLEDGMENTS}

The authors thank B. Blankleider, F. Coester, A. Stadler, J. F. J. van der Brand, and R. M. Woloshyn for very helpful discussions on various aspects of this work. They are grateful to R. D. McKeown for detailed information on the experiment of Refs. [19, 20]. P.U.S. thanks the Nuclear Theory Group of the University of Washington for the kind hospitality. R.-W.S. thanks the Nuclear Theory Group of the Argonne National Laboratory for hospitality. This work was funded by the Deutsche Forschungsgemeinschaft (DFG) under Contract No. Sa 247/11-1 and by the United States Department of Energy under Contract No. DE-FG0688ER40427.

\section{APPENDIX A: INCLUSIVE ELECTRON-NUCLEUS SCATTERING IN PLANE-WAVE IMPULSE APPROXIMATION}

The nuclear current tensor $W_{A}^{\mu \nu}\left(Q, P_{A}\right)$ contains all information on the nuclear target required for describing electron scattering. The approximations of PWIA can be cast into the form

$$
\begin{aligned}
& J_{A}^{\mu}(x)=\sum_{i=1}^{A} j_{N(i)}^{\mu}(x), \\
& \left|\mathbf{P}_{X} \beta_{X}\right\rangle=\mathcal{A}\left\{\left|\mathbf{p}_{x_{1}} \beta_{x_{1}}\right\rangle\left|\mathbf{P}_{A-1} s_{A-1} f_{A-1}\right\rangle\right\}, \\
& \left\langle\mathbf{p}_{x_{1}} \beta_{x_{1}} \mid \mathbf{P}_{A} s_{A}\right\rangle=0 .
\end{aligned}
$$

Approximation (A1) assumes that only one-nucleon currents contribute to the complete nuclear current. According to (A2) the complicated inelastic $A$-nucleon final state $\left|\mathbf{P}_{X} \beta_{X}\right\rangle$ contains a residual $(A-1)$-nucleon state with c.m. momentum $\mathbf{P}_{A-1}, s_{A-1}$ denoting its spin and $f_{A-1}$ its further discrete quantum numbers and modes of internal excitation. The $(A-1)$-nucleon state $\left|\mathbf{P}_{A-1} s_{A-1} f_{A-1}\right\rangle$ is itself fully antisymmetrized. All inelasticities are produced on the nucleon when the current acts on a single nucleon. The inelastic onenucleon states are denoted by $\left|\mathbf{p}_{x_{1}} \beta_{x_{1}}\right\rangle, \mathbf{p}_{x_{1}}$ being its c.m. momentum, $\beta_{x_{1}}$ describing all discrete quantum numbers as well as its inelastic excitation. There is no interaction between the inelastic one-nucleon and the spectator $(A-1)$-nucleon states. The inelastic one-nucleon states may be reached from any of the identical nucleons in the nucleus according to Eq. (A1). This is the reason for the antisymmetrizer $\mathcal{A}=(1 / \sqrt{A})\left(1-\sum_{i=2}^{A} P_{1 i}\right)$ in Eq. (A2), $P_{1 i}$ being the permutation operator of nucleons 1 and $i$. Approximation (A3) yields the incoherence of the scattering process: The nucleon knocked out by the electron is also in an elastic one-nucleon state so different from a bound nucleon that its overlap with a spectator nucleon vanishes. In fact, approximation (A3) is exact for inelastic nucleonic excitations.

The current matrix elements are needed in the combination

$$
\begin{aligned}
\sum_{\beta_{X}} \int d^{3} P_{X}\left\langle\mathbf{P}_{A} n_{A}\left|J_{A}^{\mu}(0)\right| \mathbf{P}_{X} \beta_{X}\right\rangle\left\langle\mathbf{P}_{X} \beta_{X}\left|J_{A}^{v}(0)\right| \mathbf{P}_{A} n_{A}\right\rangle & \\
=A \sum_{\beta_{x_{1}}} \int d^{3} p_{x_{1}} \sum_{s_{A-1} f_{A-1}} \int d^{3} P_{A-1}\left\langle\mathbf { P } _ { A } n _ { A } \left| j j_{N(1)}^{\mu}\left\{\left|\mathbf{p}_{x_{1}} \beta_{x_{1}}\right\rangle\left|\mathbf{P}_{A-1} s_{A-1} f_{A-1}\right\rangle\right\}\right.\right. & \times\left\{\left\langle\mathbf{p}_{x_{1}} \beta_{x_{1}}\left|\left\langle\mathbf{P}_{A-1} s_{A-1} f_{A-1}\right|\right\} j_{N(1)}^{v} \mid \mathbf{P}_{A} n_{A}\right\rangle(\mathbf{A} 4)\right.
\end{aligned}
$$

for the nuclear current tensor; they are evaluated under the assumptions (A1)-(A3) in Appendix A of Ref. [6]; matrix elements of the single-nucleon current are rewritten as

$$
\begin{aligned}
\left\{\left\langle\mathbf{p}_{x_{1}} \beta_{x_{1}}\right|\right. & \left.\left\langle\mathbf{P}_{A-1} s_{A-1} f_{A-1}\right|\right\} j_{N(1)}^{v}(0)\left|\mathbf{P}_{A} n_{A}\right\rangle \\
& =\sum_{t_{N}} \sum_{s_{N}} \int d^{3} p_{N}\left\langle\mathbf{p}_{x_{1}} \beta_{x_{1}}\left|j_{N(1)}^{v}(0)\right| \mathbf{p}_{N} s_{N} t_{N}\right\rangle \delta\left(\mathbf{p}_{N}+\mathbf{P}_{A-1}-\mathbf{P}_{A}\right)\left\langle\mathbf{p}_{N} s_{N} t_{N},\left(\mathbf{P}_{A}-\mathbf{p}_{N}\right) s_{A-1} f_{A-1} \mid \mathbf{P}_{A} n_{A}\right\rangle,
\end{aligned}
$$

where $s_{N}$ and $t_{N}$ stand for the spin and isospin of the nucleon. The subscript (1) is dropped in $j_{N(1)}^{v}(0)$ from now on.

The nuclear current tensor $W_{A}^{\mu \nu}\left(Q, P_{A}\right)$ is formed according to Eq. (2.1), i.e., 


$$
\left\langle n_{A}\left|W_{A}^{\mu \nu}\left(Q, P_{A}\right)\right| n_{A}\right\rangle=\sum_{s_{A} s_{A}^{\prime}}\left\langle n_{A} \mid s_{A}^{\prime}\right\rangle\left\langle s_{A}^{\prime}\left|W_{A}^{\mu \nu}\left(Q, P_{A}\right)\right| s_{A}\right\rangle\left\langle s_{A} \mid n_{A}\right\rangle,
$$

where

$$
\begin{aligned}
\left\langle s_{A}^{\prime}\left|W_{A}^{\mu v}\left(Q, P_{A}\right)\right| s_{A}\right\rangle & \\
=(2 \pi)^{6} \frac{P_{A}^{0}}{m_{A}} \sum_{t_{N}} \sum_{s_{N} s_{N}^{\prime}} \int d^{3} p_{N} \sum_{s_{A-1} f_{A-1}} & \left(\sum_{\beta_{x_{1}}} \int d^{3} p_{x_{1}}\left\langle\mathbf{p}_{N} s_{N}^{\prime} t_{N}\left|j_{N}^{\mu}(0)\right| \mathbf{p}_{x_{1}} \beta_{x_{1}}\right\rangle\right. \\
& \left.\times \delta^{4}\left(p_{x_{1}}+P_{A-1}-Q-P_{A}\right)\left\langle\mathbf{p}_{x_{1}} \beta_{x_{1}}\left|j_{N}^{v}(0)\right| \mathbf{p}_{N} s_{N} t_{N}\right\rangle\right) \\
\times & {\left[A\left\langle\mathbf{P}_{A} s_{A}^{\prime} \mid \mathbf{p}_{N} s_{N}^{\prime} t_{N},\left(\mathbf{P}_{A}-\mathbf{p}_{N}\right) s_{A-1} f_{A-1}\right\rangle\right.} \\
& \left.\times\left\langle\mathbf{p}_{N} s_{N} t_{N},\left(\mathbf{P}_{A}-\mathbf{p}_{N}\right) s_{A-1} f_{A-1} \mid \mathbf{P}_{A} s_{A}\right\rangle\right] .
\end{aligned}
$$

In the nuclear c.m. system the state $\left|n_{A}\right\rangle$ of general polarization is expanded in terms of spin states $\left|s_{A}\right\rangle$ with respect to the $z$ axis as quantization axis, i.e., $\left|n_{A}\right\rangle=\sum_{s_{A}}\left|s_{A}\right\rangle\left\langle s_{A} \mid n_{A}\right\rangle$. The momentum transfer to the nucleus $Q$ differs from the momentum transfer $Q_{N}=p_{x_{1}}-p_{N}$ to the nucleon since $P_{A-1}+p_{N} \neq P_{A}$. The $\delta$ function of energy conservation depends on the energy $E_{A}$ of the initial nuclear ground state through $P_{A}^{0} \simeq \sqrt{A^{2} m_{N}^{2}+\mathbf{P}_{A}^{2}}+E_{A}$, and on the internal excitation energy $e_{A-1}\left(f_{A-1}\right)$ of the residual $(A-1)$-nucleon system through

$$
P_{A-1}^{0}\left(e_{A-1}\left(f_{A-1}\right)\right) \simeq \sqrt{(A-1)^{2} m_{N}^{2}+\left(\mathbf{P}_{A}-\mathbf{p}_{N}\right)^{2}}+e_{A-1}\left(f_{A-1}\right),
$$

$E_{A-1}$ being the minimal energy of excitation, i.e., $E_{A-1}=\min \left\{e_{A-1}\left(f_{A-1}\right)\right\}$. We therefore rewrite the energy $\delta$ function in Eq. (A6b) by

$$
\begin{aligned}
& \delta\left(p_{x_{1}}^{0}+P_{A-1}^{0}\left(e_{A-1}\left(f_{A-1}\right)\right)-Q^{0}-P_{A}^{0}\right)=\int_{-E_{A}+E_{A-1}}^{\infty} d E \delta\left(E+E_{A}-e_{A-1}\left(f_{A-1}\right)\right) \delta\left(p_{x_{1}}^{0}+P_{A-1}^{0}\left(E+E_{A}\right)-Q^{0}-P_{A}^{0}\right) \\
& =\int_{-E_{A}+E_{A-1}}^{\infty} d E \delta\left(E+E_{A}-e_{A-1}\left(f_{A-1}\right)\right) \delta\left(p_{x_{1}}^{0}-Q_{N}^{0}\left(E, \mathbf{p}_{N}\right)-p_{N}^{0}\right),
\end{aligned}
$$

introducing explicitly the momentum transfer $Q_{N}$ to the nucleon,

$$
Q_{N}\left(E, \mathbf{p}_{N}\right)=\left(Q^{0}+P_{A}^{0}-P_{A-1}^{0}\left(E+E_{A}\right)-p_{N}^{0}, \mathbf{Q}\right) \text {, }
$$

which takes in the nuclear c.m. system, i.e., for $P_{A}^{0}=m_{A}=A m_{N}+E_{A}$, the explicit form of Eq. (3.10). The nuclear current tensor then becomes

$$
\begin{aligned}
& \left\langle s_{A}^{\prime}\left|W_{A}^{\mu v}\left(Q, P_{A}\right)\right| s_{A}\right\rangle \\
& =\sum_{t_{N}} \sum_{s_{N} s_{N}^{\prime}} \int d^{3} p_{N} \frac{m_{N}}{p_{N}^{0}} \int_{-E_{A}+E_{A-1}}^{\infty} d E\left[(2 \pi)^{6} \frac{p_{N}^{0}}{m_{N}} \sum_{\beta_{x_{1}}} \int d^{3} p_{x_{1}}\left\langle\mathbf{p}_{N} s_{N}^{\prime} t_{N}\left|j_{N}^{\mu}(0)\right| \mathbf{p}_{x_{1}} \beta_{x_{1}}\right\rangle \delta^{4}\left(p_{x_{1}}-Q_{N}\left(E, \mathbf{p}_{N}\right)-p_{N}\right)\right. \\
& \left.\times\left\langle\mathbf{p}_{x_{1}} \beta_{x_{1}}\left|j_{N}^{v}(0)\right| \mathbf{p}_{N} s_{N} t_{N}\right\rangle\right] \\
& \times\left\{A \frac{P_{A}^{0}}{m_{A}} \sum_{s_{A-1} f_{A-1}} \delta\left(E+E_{A}-e_{A-1}\left(f_{A-1}\right)\right)\left\langle\mathbf{P}_{A} s_{A}^{\prime} \mid \mathbf{p}_{N} s_{N}^{\prime} t_{N}\left(\mathbf{P}_{A}-\mathbf{p}_{N}\right) s_{A-1} f_{A-1}\right\rangle\right. \\
& \left.\times\left\langle\mathbf{p}_{N} s_{N} t_{N}\left(\mathbf{P}_{A}-\mathbf{p}_{N}\right) s_{A-1} f_{A-1} \mid \mathbf{P}_{A} s_{A}\right\rangle\right) \\
& \left\langle s_{A}^{\prime}\left|W_{A}^{\mu \nu}\left(Q, P_{A}\right)\right| s_{A}\right\rangle=\sum_{t_{N}} \sum_{s_{N} s_{N}^{\prime}} \int d^{3} p_{N} \frac{m_{N}}{p_{N}^{0}} \int_{-E_{A}+E_{A-1}}^{\infty} d E\left\langle s_{N}^{\prime}\left|W_{N\left(t_{N}\right)}^{\mu \nu}\left(Q_{N}, p_{N}\right)\right| s_{N}\right\rangle\left\langle s_{N} s_{A}^{\prime}\left|S\left(\mathbf{p}_{N} E t_{N}\right)\right| s_{N}^{\prime} s_{A}\right\rangle
\end{aligned}
$$

and is therefore the convolution between the nucleonic current tensor $W_{N\left(t_{N}\right)}^{\mu v}\left(Q_{N}, p_{N}\right)$ and the spectral function $S\left(\mathrm{p}_{N} E t_{N}\right)$ which contains the nuclear structure information. Equation (A9) proves the spin dependence (3.1) for the nuclear current tensor in PWIA. 


\section{APPENDIX B: SPIN DEPENDENCE OF NUCLEONIC CURRENT TENSOR}

The spin dependence of the nucleonic current tensor is derived in two steps. First, an identity for the positive-energy Dirac spinors is given. Second, that identity is used to obtain the spin dependence of the nucleonic current tensor.

\section{Identity for general positive-energy Dirac spinors}

$$
\begin{aligned}
& u(\mathrm{p} s) \bar{u}\left(\mathbf{p} s^{\prime}\right)=\frac{1}{2}\left\langle\alpha_{s^{\prime}}\left|1+\gamma_{5^{\delta}}{ }^{\prime}(\boldsymbol{\sigma})\right| \alpha_{s}\right\rangle(\not p+m), \\
& s(\sigma):=\left[\frac{p \cdot \boldsymbol{\sigma}}{m}, \sigma+\frac{\mathbf{p} \cdot \boldsymbol{\sigma}}{m\left(m+p^{0}\right)} \mathbf{p}\right) .
\end{aligned}
$$

The identity (B1a) is to be proven; it is an operator in the space of $\gamma$ matrices and a matrix element with respect to the orthonormal Pauli spinors $\left|\alpha_{s}\right\rangle$, defined by $\boldsymbol{\sigma} \hat{\mathbf{n}}_{R_{1}}\left|\alpha_{s}\right\rangle=s\left|\alpha_{s}\right\rangle$ with $s= \pm 1$ for a general direction $\hat{\mathbf{n}}_{R_{1}}$ of the spinor polarization. The Dirac matrices are used in the parametrization of Ref. [24]. The positive-energy spinors for a particle of mass $m$ are taken in the form

$$
u(\mathrm{p} s)=\sqrt{m+p^{0}}\left(\begin{array}{c}
I \\
\frac{\sigma \cdot \mathrm{p}}{m+p^{0}}
\end{array}\right)\left|\alpha_{s}\right\rangle
$$

and satisfy the simultaneous eigenvalue problems

$$
\begin{aligned}
& \not p u(\mathrm{p} s)=m u(\mathrm{p} s), \\
& \gamma_{5} h_{1} u(\mathrm{p} s)=s u(\mathrm{p} s),
\end{aligned}
$$

with the four-dimensional polarization vector

$$
n_{1}=\left(\frac{\mathbf{p} \cdot \hat{\mathbf{n}}_{R_{1}}}{m}, \hat{\mathbf{n}}_{R_{1}}+\frac{\mathbf{p} \cdot \hat{\mathbf{n}}_{R_{1}}}{m\left(m+p^{0}\right)} \mathbf{p}\right)
$$

and $p^{2}=m^{2}, s= \pm 1$. We note that the spin-diagonal form of the identity $u(\mathrm{p} s) \bar{u}(\mathrm{p} s)=\frac{1}{2}\left(1+\gamma_{5} s \hbar_{1}\right)(\not p+m)$ is standard. Novel as its general spin structure.

For the proof of the identity a complete set of three-dimensional orthonormal basis vectors $\left(\widehat{\mathbf{n}}_{R_{1}}, \widehat{\mathbf{n}}_{R_{2}}, \widehat{\mathbf{n}}_{R_{3}}\right)$ is chosen, the first one being the polarization vector in the particle's rest frame. The Lorentz transformation $\Lambda$ transforms restframe vectors to the frame in which the particle has four-momentum $p$, i.e., $p=\Lambda p_{R}$ with $p_{R}=(m, 0)$ and $n_{i}=\Lambda n_{R_{i}}$ with $n_{R_{i}}=\left(0, \widehat{\mathbf{n}}_{R_{i}}\right)$. The explicit form for $n_{1}$ is given in Eq. (B3c) and is representative for all three vectors $n_{i}$. The corresponding boost of the spinors is $S$, i.e., $u(\mathrm{p} s)=S u\left(\mathrm{p}_{R} s\right)$, where $S$ has the standard properties $S^{-1}=\gamma_{0} S^{+} \gamma_{0}$, $S a S^{-1}=\gamma^{v}(\Lambda a)_{v}$, and $S \gamma_{5} S^{-1}=\gamma_{5}$. The proof of the identity then follows:

$$
\begin{aligned}
u(\mathrm{p} s) \bar{u}\left(\mathrm{p} s^{\prime}\right) & =S\left|\alpha_{s}\right\rangle\left\langle\alpha_{s^{\prime}}\right|\left[\gamma^{0} m+m\right] S^{-1} \\
& =S \frac{1}{2}\left[\delta_{s s^{\prime}}+\sigma \hat{\mathbf{n}}_{R_{1}} s \delta_{s s^{\prime}}+\boldsymbol{\sigma}\left(\widehat{\mathbf{n}}_{R_{2}}+i s \widehat{\mathbf{n}}_{R_{3}}\right) \delta_{s,-s^{\prime}}\right]\left[\not \phi_{R}+m\right] S^{-1} \\
& =S \frac{1}{2}\left[\delta_{s s^{\prime}}-\gamma_{0} \gamma_{5} \gamma\left(\widehat{\mathbf{n}}_{R_{1}} s \delta_{s s^{\prime}}+\widehat{\mathbf{n}}_{R_{2}} \delta_{s,-s^{\prime}}+\widehat{\mathbf{n}}_{R_{3}} i s \delta_{s,-s^{\prime}}\right)\right]\left[\not \phi_{R}+m\right] S^{-1} \\
& =S \frac{1}{2}\left[\delta_{s s^{\prime}}+\gamma_{5}\left(n_{R_{1}} s \delta_{s s^{\prime}}+n_{R_{2}} \delta_{s,-s^{\prime}}+n_{R_{3}} i s \delta_{s,-s^{\prime}}\right)\right]\left[\not p_{R}+m\right] S^{-1} \\
& =\frac{1}{2}\left[\delta_{s s^{\prime}}+\gamma_{5}\left(n_{1} s \delta_{s s^{\prime}}+n_{2} \delta_{s,-s^{\prime}}+n_{3} i s \delta_{s,-s^{\prime}}\right)\right][\not p+m] \\
& =\frac{1}{2}\left[\delta_{s s^{\prime}}+\gamma_{5}\left\langle\alpha_{s^{\prime}}\left|\sum_{i} n_{i} \sigma \hat{\mathbf{n}}_{R_{i}}\right| \alpha_{s}\right\rangle\right)[\not p+m] \\
& =\frac{1}{2}\left\langle\alpha_{s^{\prime}}\left|1+\gamma_{5} \delta(\sigma)\right| \alpha_{s}\right\rangle[\not p+m] .
\end{aligned}
$$

In the last step the completeness of the three-dimensional basis states is used.

\section{Derivation of the spin dependence of the nucleonic current tensor}

The current tensor $W_{N\left(t_{N}\right)}^{\mu \nu}\left(Q_{N}, p_{N}\right)$ of a nucleon with mass $m_{N}$, isospin $t_{N}$, four-momentum $p_{N}$, and polarization $n_{N}$ is defined as 


$$
\left\langle n_{N}\left|W_{N\left(t_{N}\right)}^{\mu v}\left(Q_{N}, p_{N}\right)\right| n_{N}\right\rangle=\sum_{s_{N} s_{N}^{\prime}}\left\langle n_{N} \mid s_{N}^{\prime}\right\rangle\left\langle s_{N}^{\prime}\left|W_{N\left(t_{N}\right)}^{\mu v}\left(Q_{N}, p_{N}\right)\right| s_{N}\right\rangle\left\langle s_{N} \mid n_{N}\right\rangle
$$

where

$\left\langle s_{N}^{\prime}\left|W_{N\left(t_{N}\right)}^{\mu v}\left(Q_{N}, p_{N}\right)\right| s_{N}\right\rangle=(2 \pi)^{6} \frac{p_{N}^{0}}{m_{N}} \sum_{\beta_{x}} \int d^{3} p_{x}\left\langle\mathbf{p}_{N} s_{N}^{\prime} t_{N}\left|j_{N}^{\mu}(0)\right| \mathbf{p}_{x} \beta_{x}\right\rangle \delta^{4}\left(p_{x}-Q_{N}-p_{N}\right)\left\langle\mathbf{p}_{x} \beta_{x}\left|j_{N}^{v}(0)\right| \mathbf{p}_{N} s_{N} t_{N}\right\rangle$

The initial state $\left|\mathbf{p}_{N} n_{N} t_{N}\right\rangle$ is given by the polarization-dependent spinor $u$ ( $\left.\mathbf{p}_{N} n_{N}\right)$ of the form (B2), whose isospin dependence is suppressed and whose corresponding Pauli spinor in the nucleonic rest frame $\left|\alpha_{N}\right\rangle=\sum_{s_{N}}\left|s_{N}\right\rangle\left\langle s_{N} \mid n_{N}\right\rangle$ with $\sigma_{N} \hat{\mathbf{n}}_{N}\left|\alpha_{N}\right\rangle=\left|\alpha_{N}\right\rangle$ can be expanded into spin basis states $\left|s_{N}\right\rangle$ with the $z$ axis as direction for the quantization axis. The tensor $W_{N\left(t_{N}\right)}^{\mu \nu}\left(Q_{N}, p_{N}\right)$ is considered an operator in that two-dimensional spin space. The initial spinors $u\left(\mathbf{p}_{N} s_{N}\right) \bar{u}\left(\mathbf{p}_{N} s_{N}^{\prime}\right)$ in Eq. (B5b) yield the polarization part of the complete tensor. The whole Lorentz structure of the current tensor is independent of $u\left(\mathbf{p}_{N} s_{N}\right) \bar{u}\left(\mathbf{p}_{N} s_{N}^{\prime}\right)$. Introducing nucleonic structure functions, i.e., $W_{1}^{N\left(t_{N}\right)}, W_{2}^{N\left(t_{N}\right)}$, $G_{1}^{N\left(t_{N}\right)}$, and $G_{2}^{N\left(t_{N}\right)}$ and using the general identity (B1a) for the particular spinors $u\left(\mathbf{p}_{N} s_{N}\right) \bar{u}\left(\mathbf{p}_{N} s_{N}^{\prime}\right)$, the nucleonic current tensor takes the form

$$
\begin{aligned}
& \left\langle s_{N}^{\prime}\left|W_{N\left(t_{N}\right)}^{\mu \nu}\left(Q_{N}, p_{N}\right)\right| s_{N}\right\rangle=\left\langle s_{N}^{\prime}\right|\left(\frac{Q_{N}^{\mu} Q_{N}^{v}}{Q_{N}^{2}}-g^{\mu \nu}\right) W_{1}^{N\left(t_{N}\right)}\left(Q_{N}^{2}, Q_{N} \cdot p_{N} / m_{N}\right)+\frac{\widetilde{p}_{N}^{\mu} \widetilde{p}_{N}^{v}}{m_{N}^{2}} W_{2}^{N\left(t_{N}\right)}\left(Q_{N}^{2}, Q_{N} \cdot p_{N} / m_{N}\right) \\
& +i \epsilon^{\mu \nu \alpha \beta} Q_{N \alpha}\left[s_{\beta}\left(\sigma_{\mathrm{N}}\right) \frac{G_{1}^{N\left(t_{N}\right)}\left(Q_{N}^{2}, Q_{N} \cdot p_{N} / m_{N}\right)}{m_{N}}\right. \\
& \left.+\left\{\left(Q_{N} \cdot p_{N}\right) s_{\beta}\left(\sigma_{\mathrm{N}}\right)-\left[Q_{N} \cdot s\left(\sigma_{\mathrm{N}}\right)\right] p_{N \beta}\right] \frac{G_{2}^{N\left(t_{N}\right)}\left(Q_{N}^{2}, Q_{N} \cdot p_{N} / m_{N}\right)}{m_{N}^{3}}\right]\left|s_{N}\right\rangle,
\end{aligned}
$$

with

$$
\begin{aligned}
& \widetilde{p}_{N}:=p_{N}-\frac{\left(Q_{N} \cdot p_{n}\right)}{Q_{N}^{2}} Q_{N}, \\
& s\left(\sigma_{\mathbf{N}}\right):=\left[\frac{\mathbf{p}_{N} \cdot \boldsymbol{\sigma}_{\mathbf{N}}}{m_{N}}, \boldsymbol{\sigma}_{N}+\frac{\mathbf{p}_{N} \cdot \boldsymbol{\sigma}_{\mathbf{N}}}{m_{N}\left(m_{N}+p_{N}^{0}\right)} \mathbf{p}_{N}\right] .
\end{aligned}
$$

The nucleonic polarization state is defined as $\left|\alpha_{N}\right\rangle$ in the nucleonic rest frame for Eq. (B5a). - The Lorentz structure of the corresponding polarization vector $n_{N}$ is carried by the Lorentz-boosted spin operator $s\left(\sigma_{N}\right)$ in Eq. (B6c). The derived spin structure of the nucleonic current tensor is used in Sec. III.

\section{APPENDIX C: SPIN-DEPENDENT SPECTRAL FUNCTION FOR THE THREE-NUCLEON SYSTEM}

This Appendix calculates the spin-dependent spectral function of Eq. (3.4) for the three-nucleon system. The target ground state is in its c.m. system $\left|\mathbf{P}_{A}=0 s_{A} t_{A}\right\rangle$ identified with the trinucleon bound state $\left|\Psi_{B} s_{A} t_{A}\right\rangle$ of energy $E_{A}=E_{B}$ in Ref. [15]. Reference [15] obtains that state by solving the Faddeev equations in momentum space; only the solution for the purely nucleonic two-body Paris potential is used in this paper. In contrast to the main text, the dependence of the bound state on the isospin projection $t_{A}$, i.e., $t_{A}=\frac{1}{2}$ for ${ }^{3} \mathrm{He}$ and $t_{A}=-\frac{1}{2}$ for ${ }^{3} \mathrm{H}$, is made explicit and will also be kept for the spectral function in the larger part of this Appendix. The bound state is decomposed into momentumspace basis states of definite partial-wave characteristics, i.e.,

$$
\left|\mathbf{P}_{A}=0 s_{A} t_{A}\right\rangle=\sum_{L S I T} \sum_{l j} \int p^{2} d p q^{2} d q\left|p q\left[(L S) I\left(l \frac{1}{2}\right) j\right] \not \partial s_{A} ;\left(T \frac{1}{2}\right) \mathcal{T} t_{A}\right\rangle\left\langle p q\left[(L S) I\left(l \frac{1}{2}\right) j\right] \mathcal{\partial} ;\left(T \frac{1}{2}\right) \mathcal{T} \mid \Psi_{B}\right\rangle
$$

The Jacobi momenta $\mathbf{p}$ and $\mathbf{q}$ as well as all discrete quantum numbers are defined in Fig. 14. Due to rotational invariance and due to isospin independence, the expansion coefficients are independent of the angular momentum projection $s_{A}$ and of the isospin projection $t_{A}$. The trinucleon ground state has the total angular momentum and isospin $\frac{1}{2}$, i.e., $\mathscr{J}=\mathcal{T}=\frac{1}{2}$, and positive parity, i.e., $(-1)^{L+l}=1$. Reference [15] gives that state $\left|\Psi_{B}\right\rangle$ on 22 mesh points for its $p$ dependence and on 20 mesh points for its $q$ dependence.

The correlated states $\left|\mathbf{P}_{A-1} s_{A-1} f_{A-1}\right\rangle$ of the residual $(A-1)$ nucleus are tensor products of states $\left|\mathbf{P}_{A-1}\right\rangle$ describ- 
ing the $(A-1)$ c.m. motion and of states $\left|s_{A-1} f_{A-1}\right\rangle$ describing the internal motion. In the three-nucleon system the latter states are to be identified with the deuteron $\left|d I_{z} T_{z}\right\rangle$ of energy $e_{A-1}\left(f_{A-1}\right)=e_{d}$, i.e.,

$$
\left|d I_{z} T_{z}\right\rangle=\sum_{L=0,2} \sum_{S I T} \int p^{2} d p\left|p(L S) I I_{z} T T_{z}\right\rangle \delta_{S 1} \delta_{I 1} \delta_{T 0}\langle p L \mid d\rangle
$$

and with the $\delta$-function-normalized scattering states with outgoing-wave boundary conditions $\left|\psi^{+}\left(\mathbf{k}_{f}\right) S_{f} S_{f z} T_{f} T_{f z}\right\rangle$ of energy $e_{A-1}\left(f_{A-1}\right)=k_{f}^{2} / m_{N}$, i.e.,

$\left|\psi^{+}\left(\mathbf{k}_{f}\right) S_{f} S_{f z} T_{f} T_{f z}\right\rangle=\sum_{L S I I_{z} T T_{z}} \int p^{2} d p\left|p(L S) I I_{z} T T_{z}\right\rangle \delta_{S S_{f}} \delta_{T T_{f}} \delta_{T_{z} T_{f z}} \sum_{L^{\prime} L_{z}^{\prime}} Y_{L^{\prime} L_{z}^{\prime}}^{*}\left(\hat{\mathbf{k}}_{f}\right)\left\langle L^{\prime} L_{z}^{\prime} S_{f} S_{f z} \mid I I_{z}\right\rangle\left\langle p L \mid \psi^{S I T}\left(k_{f}\right) L^{\prime}\right\rangle$.

Equations (C2) and (C3) give the momentum-space partial-wave decomposition for those two-nucleon states of internal motion. The two-nucleon basis states are those of Fig. 14, already required for the definition of the three-nucleon basis states.

The plane-wave state of the knocked-out nucleon is given in terms of the spectator momentum and the corresponding discrete quantum numbers of Fig. 14, i.e.,

$$
\left|\mathbf{p}_{N} s_{N} t_{N}\right\rangle=\sum_{l j} \sum_{m m_{j}} \int q^{2} d q\left|q\left(l \frac{1}{2}\right) j m_{j} \frac{1}{2} t_{N}\right\rangle \frac{\delta\left(q-p_{N}\right)}{q p_{N}} Y_{l m}^{*}\left(\hat{\mathbf{p}}_{N}\right)\left\langle\operatorname{lm} \frac{1}{2} s_{N} \mid j m_{j}\right\rangle .
$$

The spectral function is written in terms of those wave functions:

$$
\begin{aligned}
& \left\langle s_{N} s_{A}^{\prime}\left|S\left(\mathrm{p}_{N} E t_{N} t_{A}\right)\right| s_{N}^{\prime} s_{A}\right\rangle \\
& =3 \sum_{S I T} \sum_{I_{z}} \sum_{l j l^{\prime} j^{\prime}} \sum_{m m_{j} m^{\prime} m_{j}^{\prime}} Y_{l m}\left(\widehat{\mathbf{p}}_{N}\right) Y_{l^{\prime} m^{\prime}}^{*}\left(\widehat{\mathbf{p}}_{N}\right)\left\langle\operatorname{lm} \frac{1}{2} s_{N} \mid j m_{j}\right\rangle\left\langle l^{\prime} m^{\prime} \frac{1}{2} s_{N}^{\prime} \mid j^{\prime} m_{j}^{\prime}\right\rangle\left\langle I I_{z} j m_{j} \mid \frac{1}{2} s_{A}\right\rangle\left\langle I I_{z} j^{\prime} m_{j}^{\prime} \mid \frac{1}{2} s_{A}^{\prime}\right\rangle \\
& \times\left\{\left[\delta\left(E+E_{B}-e_{d}\right) \delta_{t_{N} t_{A}} \delta_{S 1} \delta_{I 1} \delta_{T 0}\right.\right. \\
& \times \sum_{L=0,2} \int p^{2} d p\langle d \mid p L\rangle\left\langle p p_{N}\left[(L S) I\left(l \frac{1}{2}\right) j\right] d ;\left(T \frac{1}{2}\right) \mathcal{T} \mid \Psi_{B}\right\rangle \\
& \left.\times\left[\sum_{L^{\prime}=0,2} \int p^{\prime 2} d p^{\prime}\left\langle d \mid p^{\prime} L^{\prime}\right\rangle\left\langle p^{\prime} p_{N}\left[\left(L^{\prime} S\right) I\left(l^{\prime} \frac{1}{2}\right) j^{\prime}\right] d ;\left(T \frac{1}{2}\right) \mathcal{T} \mid \Psi_{B}\right\rangle\right]^{*}\right] \\
& +\left[\frac{1}{2} k_{f} m_{N}\left|\left\langle T\left(t_{A}-t_{N}\right) \frac{1}{2} t_{N} \mid \frac{1}{2} t_{A}\right\rangle\right|^{2}\right. \\
& \times \sum_{L_{f}} \sum_{L} \int p^{2} d p\left\langle\psi^{S I T}\left(k_{f}\right) L_{f} \mid p L\right\rangle\left\langle p p_{N}\left[(L S) I\left(l \frac{1}{2}\right) j\right] d ;\left(T \frac{1}{2}\right) \mathcal{T} \mid \Psi_{B}\right\rangle \\
& \times\left\{\sum_{L^{\prime}} \int p^{\prime 2} d p^{\prime}\left\langle\psi^{S I T}\left(k_{f}\right) L_{f} \mid p^{\prime} L^{\prime}\right\rangle\right. \\
& \left.\left.\left.\times\left\langle p^{\prime} p_{N}\left[\left(L^{\prime} S\right) I\left(l^{\prime} \frac{1}{2}\right) j^{\prime}\right] \mathcal{d} ;\left(T \frac{1}{2}\right) \mathcal{T} \mid \Psi_{B}\right\rangle\right]^{*}\right] k_{f}=\sqrt{\left(E+E_{B}\right) / m_{N}}\right\} .
\end{aligned}
$$

The two- and three-body breakup contributions are additive. In the chosen normalization the three-body breakup part vanishes at $E+E_{B}=0$ for all momenta $\mathbf{p}_{N}$. The dimension of the spectral function is $\mathrm{fm}^{3} \mathrm{MeV}^{-1} . \mathrm{There}^{\mathrm{are}}$ simple isospin relations when splitting up the spectral function (C5) according to the isospin $T$ of the pair, i.e., if $S\left(\mathrm{p}_{N} E t_{N} t_{A}\right)=\sum_{T=0,1} S^{T}\left(\mathrm{p}_{N} E t_{N} t_{A}\right)$, then

$$
\begin{aligned}
& S^{T}\left(\mathrm{p}_{N} E t_{N} t_{A}\right)=S^{T}\left(\mathrm{p}_{N} E\left(-t_{N}\right)\left(-t_{A}\right)\right), \\
& S^{0}\left(\mathrm{p}_{N} E t_{N} t_{A}\right) \propto \delta_{t_{N} t_{A}}, \\
& S^{1}\left(\mathrm{p}_{N} E\left(-t_{A}\right) t_{A}\right)=2 S^{1}\left(\mathrm{p}_{N} E t_{A} t_{A}\right),
\end{aligned}
$$

provided the binding energy $E_{B}$ is taken to be the same for both nuclei ${ }^{3} \mathbf{H e}$ and ${ }^{3} \mathbf{H}$. Thus, it is sufficient to calculate the proton spectral function of ${ }^{3} \mathrm{He}$ for the two pair-isospin $T$ contributions with $t_{N}=\frac{1}{2}$ and $t_{A}=\frac{1}{2}$. The dependence on the isospin projection $t_{A}$ will therefore be omitted from now on. 
The spin-dependent spectral function also depends on the direction of $\mathbf{p}_{N}$. According to the discussion of Eq. (3.6) it can be parametrized by simple spin-independent functions of the magnitude $p_{N}=\left|\mathbf{p}_{N}\right|$ only, i.e., by $f_{0}\left(p_{N} E t_{N}\right)$, $f_{1}\left(p_{N} E t_{N}\right)$, and $f_{2}\left(p_{N} E t_{N}\right)$. Those functions follow from the multipole components $S_{\mathcal{L} M_{\mathcal{L}}}\left(p_{N} E t_{N}\right)$ of the spectral function in the form (C5):

$$
S_{\mathcal{L} M_{\mathcal{L}}}\left(p_{N} E t_{N}\right)=\int d^{2} \widehat{\mathbf{p}}_{N} Y_{\mathcal{L} M_{\mathcal{L}}}\left(\widehat{\mathbf{p}}_{N}\right) S\left(\mathbf{p}_{N} E t_{N}\right)
$$

which can be evaluated right away with the help of the standard relation

$$
Y_{l m}\left(\hat{\mathbf{p}}_{N}\right) Y_{l^{\prime} m^{\prime}}^{*}\left(\hat{\mathbf{p}}_{N}\right)=\sum_{\mathcal{L} M_{\mathcal{L}}}(-1)^{m^{\prime}}\left[\frac{(2 l+1)\left(2 l^{\prime}+1\right)(2 \mathcal{L}+1)}{4 \pi}\right]^{1 / 2}\left[\begin{array}{ccc}
l & l^{\prime} & \mathcal{L} \\
m & -m^{\prime} & M_{\mathcal{L}}
\end{array}\right]\left[\begin{array}{lll}
l & l^{\prime} & \mathcal{L} \\
0 & 0 & 0
\end{array}\right] Y_{\mathcal{L}}^{*} M_{\mathcal{L}}\left(\hat{\mathbf{p}}_{N}\right) .
$$

The functions $f_{0}\left(p_{N} E t_{N}\right), f_{1}\left(p_{N} E t_{N}\right)$, and $f_{2}\left(p_{N} E t_{N}\right)$ are computed by

$$
\begin{aligned}
& f_{0}\left(p_{N} E t_{N}\right)=\frac{1}{2}\left[\frac{1}{4 \pi}\right]^{1 / 2} \sum_{s_{N} s_{A}}\left\langle s_{N} s_{A}\left|S_{00}\left(p_{N} E t_{N}\right)\right| s_{N} s_{A}\right\rangle, \\
& f_{1}\left(p_{N} E t_{N}\right)=\frac{1}{2}\left[\frac{1}{4 \pi}\right]^{1 / 2} \sum_{s_{N} s_{A}}(-1)^{1-s_{N}-s_{A}}\left\langle s_{N} s_{A}\left|S_{00}\left(p_{N} E t_{N}\right)\right| s_{N} s_{A}\right\rangle, \\
& f_{2}\left(p_{N} E t_{N}\right)=\frac{1}{2}\left[\frac{45}{16 \pi}\right]^{1 / 2} \sum_{s_{N} s_{A}}(-1)^{1-s_{N}-s_{A}}\left\langle s_{N} s_{A}\left|S_{20}\left(p_{N} E t_{N}\right)\right| s_{N} s_{A}\right\rangle .
\end{aligned}
$$

Examples for those functions are given in Figs. 15-17. The functions $f_{1}\left(p_{N} E t_{N}\right)$ and $f_{2}\left(p_{N} E t_{N}\right)$ do not have probability interpretations and are therefore not positive definite.

The spectral function satisfies a number of sum rules which relate quantities obtained from the spectral function to quantities more directly computed from the trinucleon bound-state wave function. The observation of sum rules for the calculated spectral function indicates the numerical accuracy of its evaluation. When integrating out the energy dependence of the spectral function the spin-dependent nucleonic density $\rho\left(\mathbf{p}_{N} t_{N}\right)$, the probability for finding a nucleon with isospin $t_{N}$, with momentum $\mathbf{p}_{N}$, and with specified spin characteristics in the nucleus, results, i.e.,

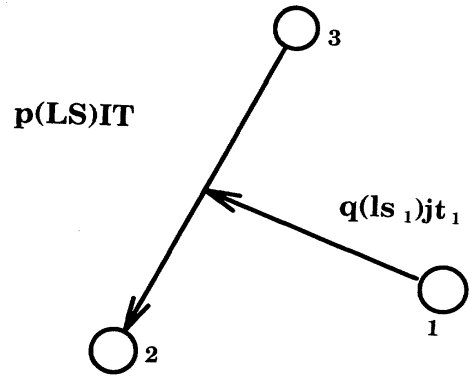

FIG. 14. Three-body Jacobi coordinates. The magnitudes of the corresponding momenta are $p$ and $q$. In the momentumspace basis states $|p q v\rangle_{1}$ the respective angular momenta $I$ and $j$ and the respective isospins $T$ and $t_{1}$ of the antisymmetrized state of nucleons 2 and 3 and of the spectator state are coupled, i.e., $\left|p q\left[(L S) I\left(l s_{1}\right) j\right] d s_{A} ;\left(T t_{1}\right) \mathcal{T} t_{A}\right\rangle_{1}$ as in Eq. (C1). The quantum numbers $L(l)$ and $S\left(s_{1}\right)$ refer to the orbital momentum and spin of the pair (spectator), $\mathcal{W}\left(s_{A}\right)$ and $\mathcal{T}\left(t_{A}\right)$ are total angular momentum (projection) and total isospin (projection) of the three-body bound state. Since all particles are nucleons, $s_{1}=t_{1}=\frac{1}{2}$. For a two-body potential with partial waves up to $I=2$ there are 18 Faddeev amplitudes in the $|p q v\rangle_{1}$ basis; for the bound state wave function $\left|\Psi_{B} s_{A} t_{A}\right\rangle$ of Eq. (C1) all channels up to $L+l \leq 8$ are kept.

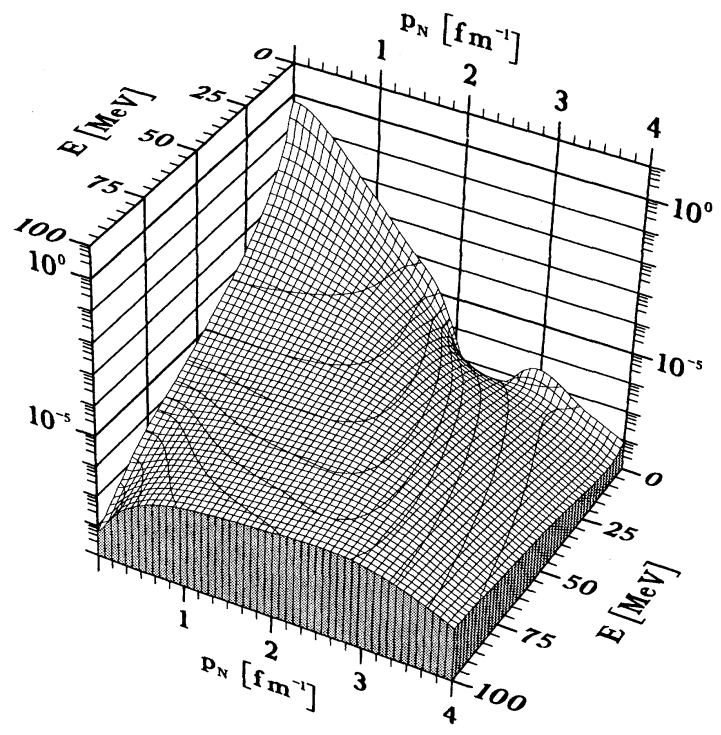

FIG. 15. Function $f_{0}\left(p_{N} E t_{N}\right)$ in units of $\left[\mathrm{fm}^{3} \mathrm{MeV}^{-1}\right]$, building up the spin-dependent spectral function according to Eq. (3.6). The contribution of the ${ }^{3} \mathrm{He}$ proton function arising from correlated nucleon pairs with isospin $T=1$ are shown for a limited energy-momentum domain in a semilog plot. 


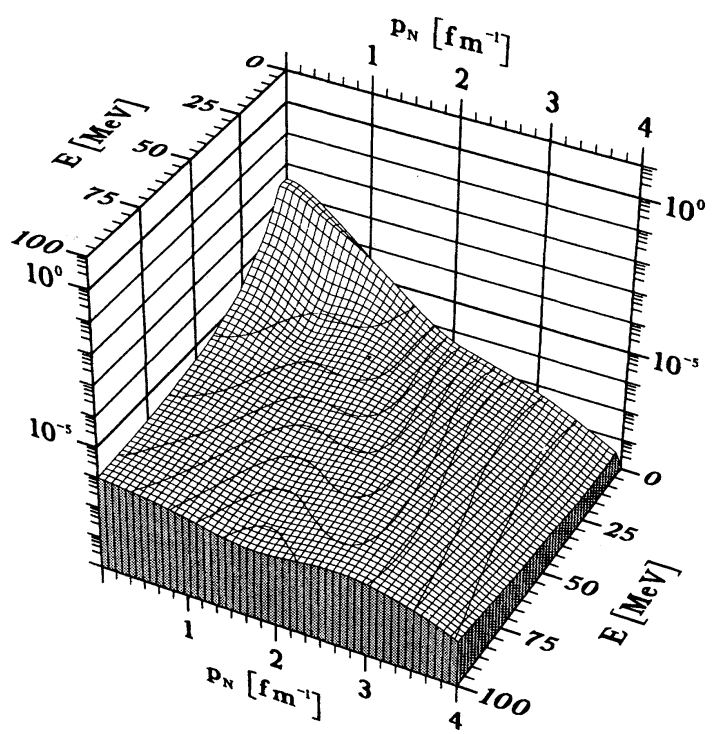

FIG. 16. Function $\left|f_{1}\left(p_{N} E t_{N}\right)\right|$ in units of $\left[\mathrm{fm}^{3} \mathrm{MeV}^{-1}\right]$, building up the spin-dependent spectral function according to Eq. (3.6). The contribution of the ${ }^{3} \mathrm{He}$ proton function arising from correlated nucleon pairs with isospin $T=0$ are shown for a limited energy-momentum domain in a semilog plot. In that domain the function happens to be negative without any change of sign. The singular contribution arising from two-body break-up is not indicated.

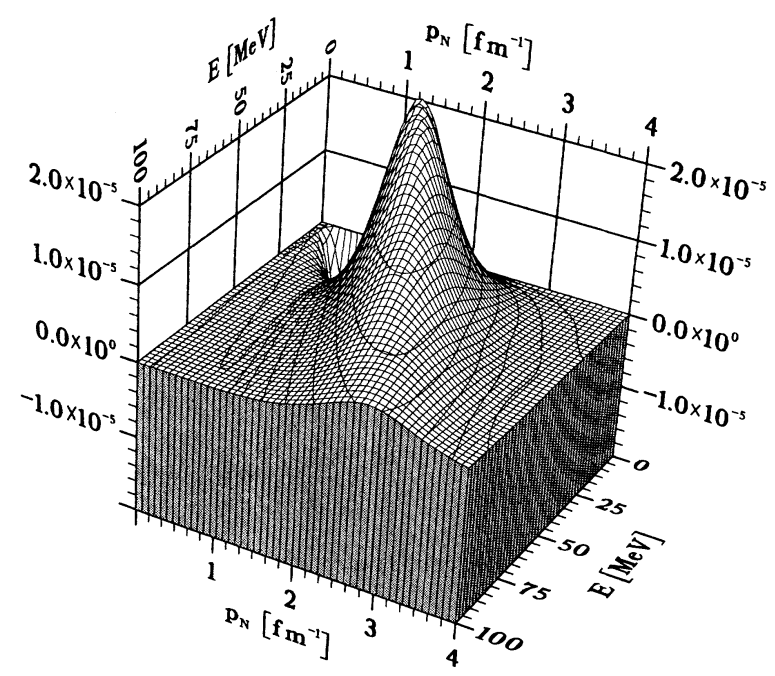

FIG. 17. Function $p_{N}^{2} f_{2}\left(p_{N} E t_{N}\right)$ in units of $[\mathrm{fm} \mathrm{MeV}-1]$, building up the spin-dependent spectral function according to Eq. (3.6). The contribution of the ${ }^{3} \mathrm{He}$ proton function arising from correlated nucleon pairs with isospin $T=1$ are shown in a limited energy-momentum domain.

$$
\begin{aligned}
\left\langle s_{N} s_{A}^{\prime}\left|\rho\left(\mathbf{p}_{N} t_{N}\right)\right| s_{N}^{\prime} s_{A}\right\rangle:=\int d E\left\langle s_{N} s_{A}^{\prime}\left|S\left(\mathbf{p}_{N} E t_{N}\right)\right| s_{N}^{\prime} s_{A}\right\rangle, \\
\left\langle s_{N} s_{A}^{\prime}\left|\rho\left(\mathbf{p}_{N} t_{N}\right)\right| s_{N}^{\prime} s_{A}\right\rangle=3 \sum_{S I T} \sum_{I_{z}} \sum_{l j l^{\prime} j^{\prime}} \sum_{m m_{j} m^{\prime} m_{j}^{\prime}} Y_{l m}\left(\hat{\mathbf{p}}_{N}\right) Y_{l^{\prime} m^{\prime}}^{*}\left(\hat{\mathbf{p}}_{N}\right)\left\langle l m \frac{1}{2} s_{N} \mid j m_{j}\right\rangle\left\langle l^{\prime} m^{\prime} \frac{1}{2} s_{N}^{\prime} \mid j^{\prime} m_{j}^{\prime}\right\rangle\left\langle I I_{z} j m_{j} \mid \frac{1}{2} s_{A}\right\rangle \\
\times\left\langle I I_{z} j^{\prime} m_{j}^{\prime} \mid \frac{1}{2} s_{A}^{\prime}\right\rangle\left|\left\langle T\left(t_{A}-t_{N}\right) \frac{1}{2} t_{N} \mid \frac{1}{2} t_{A}\right\rangle\right|^{2} \\
\times \sum_{L} \int p^{2} d p\left\langle p p_{N}\left[(L S) I\left(l \frac{1}{2}\right) j\right] \mathcal{d} ;\left(T \frac{1}{2}\right) \mathcal{T} \mid \Psi_{B}\right\rangle \\
\times\left\langle p p_{N}\left[(L S) I\left(l^{\prime} \frac{1}{2}\right) j^{\prime}\right] \mathcal{d} ;\left(T \frac{1}{2}\right) \mathcal{T} \mid \Psi_{B}\right\rangle^{*} .
\end{aligned}
$$

The nucleonic density $\rho\left(\mathbf{p}_{N} t_{N}\right)$ has the same dependence (3.6) on the nucleonic and the nuclear spins and on the direction of $\mathbf{p}_{N}$ as the full spectral function. Thus, it can be expanded into a linear combination of the spin operators $1, \sigma_{N} \sigma_{A}$, and $\left[\left(\sigma_{N} \hat{\mathbf{p}}_{N}\right)\left(\sigma_{A} \hat{\mathbf{p}}_{N}\right)-\frac{1}{3} \sigma_{N} \sigma_{A}\right]$ in the same way as the spectral function according to Eq. (3.6). We note that the spectral function $S_{\mathrm{BW}}\left(\mathrm{p}_{N} E t_{N}\right)$, introduced by Blankleider and Woloshyn in Ref. [13] and based on closure approximation, is related to that nucleonic density $\rho\left(\mathbf{p}_{N} t_{N}\right)$ by

$$
S_{\mathrm{BW}}\left(\mathbf{p}_{N} E t_{N}\right)=\delta\left(E+E_{B}-\bar{e}_{2}\left(f_{2}\right)\right) \rho\left(\mathbf{p}_{N} t_{N}\right),
$$

with the average excitation $\bar{e}_{2}\left(f_{2}\right)$ in the residual two-nucleon system taken in Ref. [13] to be of zero energy. Introducing the isospin-dependent norms

$$
\begin{aligned}
& N_{0}\left(t_{N}\right):=4 \pi \int p_{N}^{2} d p_{N} \int d E f_{0}\left(p_{N} E t_{N}\right), \\
& N_{1}\left(t_{N}\right):=4 \pi \int p_{N}^{2} d p_{N} \int d E f_{1}\left(p_{N} E t_{N}\right), \\
& N_{2}\left(t_{N}\right):=4 \pi \int p_{N}^{2} d p_{N} \int d E f_{2}\left(p_{N} E t_{N}\right),
\end{aligned}
$$

the complete integral over the spectral function for a three-nucleon bound state of polarization $n_{A}$, i.e., for $\boldsymbol{\sigma}_{\mathbf{A}} \cdot \hat{\mathbf{n}}_{A}\left|n_{A}\right\rangle=\left|n_{A}\right\rangle$, takes the form 


$$
\begin{aligned}
\int d^{3} p_{N} \int d E\left\langle n_{N} n_{A}\left|S\left(\mathbf{p}_{N} E t_{N}\right)\right| n_{N} n_{A}\right\rangle & \\
& =\frac{1}{2}\left[N_{0}\left(t_{N}\right)+N_{1}\left(t_{N}\right) \hat{\mathbf{n}}_{N} \cdot \widehat{\mathbf{n}}_{A}+N_{2}\left(t_{N}\right) \frac{1}{4 \pi} \int d^{2} \widehat{\mathbf{p}}_{N}\left[\left(\hat{\mathbf{n}}_{N} \cdot \widehat{\mathbf{p}}_{N}\right)\left(\hat{\mathbf{n}}_{A} \cdot \widehat{\mathbf{p}}_{N}\right)-\frac{1}{3} \widehat{\mathbf{n}}_{N} \cdot \widehat{\mathbf{n}}_{A}\right]\right) .
\end{aligned}
$$

Thus, $N_{0}\left(t_{N}\right)$ is the number of nucleons with isospin $t_{N}$ in the three-nucleon bound state, irrespective of nuclear and nucleonic polarization. The number of nucleons with isospin $t_{N}$ and with spin in the direction of the nuclear spin is $\frac{1}{2}\left[N_{0}\left(t_{N}\right)+N_{1}\left(t_{N}\right)\right]$, whereas $N_{1}\left(t_{N}\right)$ is the expectation value of the nucleonic spin in units of $\frac{1}{2} \hbar$. The number $N_{2}\left(t_{N}\right)$ could give information on nucleons with a particular direction of their momentum $\mathbf{p}_{N}$. However, the full angular integral, left on purpose unevaluated in Eq. (C13), is zero. Thus, $N_{2}\left(t_{N}\right)$ does not contribute to the sum rule (C13).

The spectral function is computed in the limited domain $5.1 \mathrm{MeV} \leq E \leq 200 \mathrm{MeV}$ and $0 \leq p_{N} \leq 6.5 \mathrm{fm}^{-1}$. For the prediction of quasielastic scattering data in Sec. $\mathrm{V}$ the spectral function is needed only inside that computed domain; its values are stable within $1 \%$ for all attempted improvements in the trinucleon wave function $\left|\Psi_{B}\right\rangle$, e.g., for an increase in the number of mesh points. However, the spectral function outside that computed domain will matter for predictions of deep-inelastic scattering. Technically, it already matters for sum rules, e.g., results according to Eq. (C10a) and to Eq. (C10b) differ by less than $1 \%$ for $0 \leq p_{N} \leq 2.5 \mathrm{fm}^{-1}$ except in the neighborhood of zeros, but their difference increases with $p_{N}$. The isospin-dependent norms of Eq. (C12), i.e., $N_{0}\left(t_{N}\right), N_{1}\left(t_{N}\right)$, and $N_{2}\left(t_{N}\right)$ agree in calculations from the limited spectral function $S\left(\mathbf{p}_{N} E t_{N}\right)$ and from the density $\rho\left(\mathbf{p}_{N} t_{N}\right)$ to better than $0.2 \%, 0.5 \%$, and $2.0 \%$, respectively.

\section{APPENDIX D: DIFFERENT PROCEDURES FOR EXTRACTING STRUCTURE FUNCTIONS FROM THE PWIA NUCLEAR CURRENT TENSOR}

As described in Sec. II, the nuclear current tensor has the general form

$$
\left\langle n_{A}\left|W_{A}^{\mu \nu}\left(Q, P_{A}\right)\right| n_{A}\right\rangle=\left(\frac{Q^{\mu} Q^{\nu}}{Q^{2}}-g^{\mu \nu}\right) W_{1}^{A}\left(Q^{2}, Q \cdot P_{A} / m_{A}\right)+\widetilde{P}_{A}^{\mu} \widetilde{P}_{A}^{v} \frac{W_{2}^{A}\left(Q^{2}, Q \cdot P_{A} / m_{A}\right)}{m_{A}^{2}}+i \epsilon^{\mu \nu \alpha \beta} \Delta_{\alpha \beta}\left(Q, P_{A}, n_{A}\right)
$$

(D1a)

with

$$
\Delta_{\alpha \beta}\left(Q, P_{A}, n_{A}\right)=Q_{\alpha}\left(n_{A \beta} \frac{G_{1}^{A}\left(Q^{2}, Q \cdot P_{A} / m_{A}\right)}{m_{A}}+\left[\left(Q \cdot P_{A}\right) n_{A \beta}-\left(Q \cdot n_{A}\right) P_{A \beta}\right] \frac{G_{2}^{A}\left(Q^{2}, Q \cdot P_{A} / m_{A}\right)}{m_{A}^{3}}\right)
$$

being a second-rank tensor without obvious symmetry properties. When determining the nuclear structure functions $W_{1}^{A}, W_{2}^{A}, G_{1}^{A}$, and $G_{2}^{A}$ in PWIA, contractions of the nuclear current tensor with other tensors are carried out and current conservation is used in the actual evaluation of those contractions. E.g., the extraction scheme (A) given in Eq. (3.11) does not employ longitudinal components of the current tensor, replacing them by the corresponding charge components. The nuclear structure functions of Eq. (3.11) take for their numerical analysis the final form

$$
\begin{aligned}
& W_{1}^{A}\left(Q^{2}, Q \cdot P_{A} / m_{A}\right) \\
& =\sum_{t_{N}} \int d^{3} p_{N} \frac{m_{N}}{p_{N}^{0}} \int d E\left[W_{1}^{N\left(t_{N}\right)}\left(Q_{N}^{2}, Q_{N} \cdot p_{N} / m_{N}\right)+\frac{W_{2}^{N\left(t_{N}\right)}\left(Q_{N}^{2}, Q_{N} \cdot p_{N} / m_{N}\right)}{2 m_{N}^{2}}\left|\hat{\mathbf{Q}} \times \mathbf{p}_{N}\right|^{2}\right] f_{0}\left(p_{N} E t_{N}\right), \\
& W_{2}^{A}\left(Q^{2}, Q \cdot P_{A} / m_{A}\right)=\sum_{t_{N}} \int d^{3} p_{N} \frac{m_{N}}{p_{N}^{0}} \int d E\left\{\frac{W_{2}^{N\left(t_{N}\right)}\left(Q_{N}^{2}, Q_{N} \cdot p_{N} / m_{N}\right)}{m_{N}^{2}}\left[\frac{Q^{4}}{\mathbf{Q}^{4}}\left[p_{N}^{0}-Q_{N}^{0} \frac{Q_{N} \cdot p_{N}}{Q_{N}^{2}}\right]^{2}-\frac{1}{2} \frac{Q^{2}}{\mathbf{Q}^{2}}\left|\hat{\mathbf{Q}} \times \mathbf{p}_{N}\right|^{2}\right]\right. \\
& \left.-W_{1}^{N\left(t_{N}\right)}\left(Q_{N}^{2}, Q_{N} \cdot p_{N} / m_{N}\right) \frac{Q^{2}}{\mathbf{Q}^{2}}\left[1-\frac{Q^{2}}{Q_{N}^{2}}\right]\right\} f_{0}\left(p_{N} E t_{N}\right),
\end{aligned}
$$




$$
\begin{aligned}
& G_{1}^{A}\left(Q^{2}, Q \cdot P_{A} / m_{A}\right)=\frac{m_{A}^{2}}{\mathbf{Q}^{2}} \sum_{t_{N}} \int d^{3} p_{N} \frac{m_{N}}{p_{N}^{0}} \int d E\left[\left\{G_{1}^{N\left(t_{N}\right)}\left(Q_{N}^{2}, Q_{N} \cdot p_{N} / m_{N}\right)\left[-\frac{Q^{2}}{m_{A} m_{N}} \int 1+\frac{\left(\mathbf{p}_{N} \cdot \hat{\mathbf{e}}_{1}\right)^{2}}{m_{N}\left(m_{N}+p_{N}^{0}\right)}\right]\right.\right. \\
& \left.+\frac{Q^{0}}{m_{A}} \frac{1}{m_{N}^{2}}\left[Q_{N} \cdot p_{N}-\frac{Q_{N}^{0}\left(\mathbf{p}_{N} \times \widehat{\mathbf{Q}}\right)^{2}}{m_{N}+p_{N}^{0}}\right]\right] \\
& +G_{2}^{N\left(t_{N}\right)}\left(Q_{N}^{2}, Q_{N} \cdot p_{N} / m_{N}\right)\left[-\frac{Q^{2}}{m_{A} m_{N}}\left(\frac{Q_{N} \cdot p_{N}}{m_{N}^{2}}-\frac{Q_{N}^{0}\left(\mathbf{p}_{N} \cdot \hat{\mathbf{e}}_{1}\right)^{2}}{m_{N}^{2}\left(m_{N}+p_{N}^{0}\right)}\right]\right. \\
& \left.\left.+\frac{Q^{0}}{m_{A}} \frac{Q_{N}^{2}}{m_{N}^{2}}\left[1+\frac{\left(\mathbf{p}_{N} \times \hat{\mathbf{Q}}\right)^{2}}{m_{N}\left(m_{N}+p_{N}^{0}\right)}\right]\right]\right\} \\
& \times\left[f_{1}\left(p_{N} E t_{N}\right)-\frac{1}{3} f_{2}\left(p_{N} E t_{N}\right)\right] \\
& +\left[G _ { 1 } ^ { N ( t _ { N } ) } ( Q _ { N } ^ { 2 } , Q _ { N } \cdot p _ { N } / m _ { N } ) \left[-\frac{Q^{2}}{m_{A} m_{N}} \frac{p_{N}^{0}}{m_{N}}\left(\widehat{\mathbf{p}}_{N} \cdot \widehat{\mathbf{e}}_{1}\right)^{2}\right.\right. \\
& \left.+\frac{Q^{0}}{m_{A}} \frac{1}{m_{N}^{2}}\left[Q_{N} \cdot p_{N}-Q_{N}^{0} p_{N}^{0}\left(\hat{\mathbf{p}}_{N} \times \widehat{\mathbf{Q}}\right)^{2}\right]\right] \\
& +G_{2}^{N\left(t_{N}\right)}\left(Q_{N}^{2}, Q_{N} \cdot p_{N} / m_{N}\right)\left(-\frac{Q^{2}}{m_{A} m_{N}} \frac{Q_{N}^{0}}{m_{N}}\left(\widehat{\mathbf{p}}_{N} \cdot \widehat{\mathbf{e}}_{1}\right)^{2}\right. \\
& \left.\left.\left.+\frac{Q^{0}}{m_{A}} \frac{Q_{N}^{2}}{m_{N}^{2}}\left(\widehat{\mathbf{p}}_{N} \cdot \widehat{\mathbf{Q}}\right)^{2}\right]\right] f_{2}\left(p_{N} E t_{N}\right)\right],
\end{aligned}
$$

$$
\begin{aligned}
& G_{2}^{A}\left(Q^{2}, Q \cdot P_{A} / m_{A}\right)=-\frac{m_{A}^{2}}{\mathbf{Q}^{2}} \sum_{t_{N}} \int d^{3} p_{N} \frac{m_{N}}{p_{N}^{0}} \int d E\left[\left\{G_{1}^{N\left(t_{N}\right)}\left(Q_{N}^{2}, Q_{N} \cdot p_{N} / m_{N}\right)\left[-\frac{Q^{0}}{m_{N}} \int 1+\frac{\left(\mathbf{p}_{N} \cdot \hat{\mathbf{e}}_{1}\right)^{2}}{m_{N}\left(m_{N}+p_{N}^{0}\right)}\right]\right.\right. \\
& \left.+\frac{1}{m_{N}^{2}}\left[Q_{N} \cdot p_{N}-\frac{Q_{N}^{0}\left(\mathbf{p}_{N} \times \hat{\mathbf{Q}}\right)^{2}}{m_{N}+p_{N}^{0}}\right]\right] \\
& +G_{2}^{N\left(t_{N}\right)}\left(Q_{N}^{2}, Q_{N} \cdot p_{N} / m_{N}\right)\left[-\frac{Q^{0}}{m_{N}}\left(\frac{Q_{N} \cdot p_{N}}{m_{N}^{2}}-\frac{Q_{N}^{0}\left(\mathbf{p}_{N} \cdot \widehat{\mathbf{e}}_{1}\right)^{2}}{m_{N}^{2}\left(m_{N}+p_{N}^{0}\right)}\right]\right. \\
& \left.\left.+\frac{Q_{N}^{2}}{m_{N}^{2}}\left[1+\frac{\left(\mathbf{p}_{N} \times \hat{\mathbf{Q}}\right)^{2}}{m_{N}\left(m_{N}+p_{N}^{0}\right)}\right]\right]\right\} \\
& \times\left[f_{1}\left(p_{N} E t_{N}\right)-\frac{1}{3} f_{2}\left(p_{N} E t_{N}\right)\right] \\
& +\left[G _ { 1 } ^ { N ( t _ { N } ) } ( Q _ { N } ^ { 2 } , Q _ { N } \cdot p _ { N } / m _ { N } ) \left[-\frac{Q^{0}}{m_{N}} \frac{p_{N}^{0}}{m_{N}}\left(\widehat{\mathbf{p}}_{N} \cdot \widehat{\mathrm{e}}_{1}\right)^{2}\right.\right. \\
& \left.+\frac{1}{m_{N}^{2}}\left[\left(Q_{N} \cdot p_{N}\right)-Q_{N}^{0} p_{N}^{0}\left(\widehat{\mathbf{p}}_{N} \times \widehat{\mathbf{Q}}\right)^{2}\right]\right] \\
& +G_{2}^{N\left(t_{N}\right)}\left(Q_{N}^{2}, Q_{N} \cdot p_{N} / m_{N}\right)\left[-\frac{Q^{0}}{m_{N}} \frac{Q_{N}^{0}}{m_{N}}\left[\hat{\mathbf{p}}_{N} \cdot\left(\hat{\mathbf{Q}} \times \widehat{\mathbf{e}}_{N}\right)\right]^{2}\right. \\
& \left.\left.\left.+\frac{Q_{N}^{2}}{m_{N}^{2}}\left(\widehat{\mathbf{p}}_{N} \cdot \hat{\mathbf{Q}}\right)^{2}\right]\right] f_{2}\left(p_{N} E t_{N}\right)\right] \text {. }
\end{aligned}
$$


The independence of the spin structure functions $G_{1}^{A}$ and $G_{2}^{A}$ from the polarization vector $n_{A}$ is explicit in Eqs. (D2c) and (D2d). The PWIA current tensor $W_{A}^{\mu \nu}\left(Q, P_{A}\right)$ does not satisfy current conservation. This is the reason why different extraction schemes for the nuclear structure functions yield different results. The extraction scheme (A) is based on the idea that impulse approximation is better justified for the charge than for the spatial part of the current, which usually receives substantial contributions from exchange currents. Though that idea is correct, it is not too helpful: It only fully applies to the spin-averaged longitudinal response $R_{L}$, which is given by the component $W_{A}^{00}\left(Q, P_{A}\right)$ of the nuclear current tensor according to Eq. (2.7b); in contrast, all other response functions and all structure functions also depend on spatial components $W_{A}^{i v}\left(Q, P_{A}\right)$ and $W_{A}^{\mu i}\left(Q, P_{A}\right)$ with $i=1,2$ which cannot be eliminated by the condition of current conservation. In order to explore the theoretical uncertainties inherent in the chosen extraction scheme (A), alternatives are formally derived and compared in their practical consequences for the structure functions.

As one alternative one may replace all charge components of the nuclear current tensor $W_{A}^{\mu \nu}\left(Q, P_{A}\right)$ in Eq. (2.5) by the longitudinal ones due to current conservation, i.e., $W_{A}^{0 v}\left(Q, P_{A}\right)=|Q| W_{A}^{3 v}\left(Q, P_{A}\right) / Q^{0}$ and $W_{A}^{\mu 0}\left(Q, P_{A}\right)$ $=W_{A}^{\mu 3}\left(Q, P_{A}\right)|\mathbf{Q}| / Q^{0}$, and, consequently, one may assume that only the spatial nuclear current matrix elements are given in PWIA according to Eq. (3.8). In this extraction scheme (B) the following convolution formulas result for the nuclear structure functions:

$$
\begin{gathered}
W_{1}^{A}\left(Q^{2}, Q \cdot P_{A} / m_{A}\right) \\
=\sum_{t_{N}} \int d^{3} p_{N} \frac{m_{N}}{p_{N}^{0}} \int d E\left[W_{1}^{N\left(t_{N}\right)}\left(Q_{N}^{2}, Q_{N} \cdot p_{N} / m_{N}\right)+\frac{W_{2}^{N\left(t_{N}\right)}\left(Q_{N}^{2}, Q_{N} \cdot p_{N} / m_{N}\right)}{2 m_{N}^{2}}\left|\hat{\mathbf{Q}} \times \mathbf{p}_{N}\right|^{2}\right] f_{0}\left(p_{N} E t_{N}\right), \\
W_{2}^{A}\left(Q^{2}, Q \cdot P_{A} / m_{A}\right) \\
=\sum_{t_{N}} \int d^{3} p_{N} \frac{m_{N}}{p_{N}^{0}} \int d E\left\{\frac{W_{2}^{N\left(t_{N}\right)}\left(Q_{N}^{2}, Q_{N} \cdot p_{N} / m_{N}\right)}{m_{N}^{2}}\left[\frac{Q^{4}}{\left(Q^{0}\right)^{2} \mathbf{Q}^{2}}\left[\mathbf{p}_{N} \cdot \hat{\mathbf{Q}}-|\mathbf{Q}| \frac{Q_{N} \cdot p_{N}}{Q_{N}^{2}}\right]^{2}-\frac{1}{2} \frac{Q^{2}}{\mathbf{Q}^{2}}\left|\hat{\mathbf{Q}} \times \mathbf{p}_{N}\right|^{2}\right]\right. \\
\left.-W_{1}^{N\left(t_{N}\right)}\left(Q_{N}^{2}, Q_{N} \cdot p_{N} / m_{N}\right) \frac{Q^{2}}{\mathbf{Q}^{2}}\left[1-\frac{Q^{2}}{Q_{N}^{2}}\left[\frac{Q_{N}^{0}}{Q^{0}}\right]^{2}\right]\right\} f_{0}\left(p_{N} E t_{N}\right),
\end{gathered}
$$

$$
\begin{aligned}
& G_{1}^{A}\left(Q^{2}, Q \cdot P_{A} / m_{A}\right) \\
& =\frac{m_{A}^{2}}{\mathbf{Q}^{2}} \sum_{t_{N}} \int d^{3} p_{N} \frac{m_{N}}{p_{N}^{0}} \int d E\left[-\frac{Q^{2}}{m_{A}} \frac{Q_{N}^{0}}{Q^{0}} \frac{1}{n_{A}^{1}} g^{1 \alpha}+\frac{Q^{0}}{m_{A}} \frac{1}{n_{a}^{3}}\left(Q_{N}^{0} g^{3 \alpha}-|\mathbf{Q}| g^{0 \alpha}\right)\right] \\
& \times\left(\mathcal{S}_{\alpha}\left(\mathbf{p}_{N} E t_{N} \widehat{\mathbf{n}}_{A}\right) \frac{G_{1}^{N}\left(Q_{N}^{2}, Q_{N} \cdot p_{N} / m_{N}\right)}{m_{N}}\right. \\
& \left.+\left\{\left(Q_{N} \cdot p_{N}\right) \mathcal{S}_{\alpha}\left(\mathbf{p}_{N} E t_{N} \widehat{\mathbf{n}}_{A}\right)-\left[Q_{N} \cdot \mathcal{S}\left(\mathbf{p}_{N} E t_{N} \widehat{\mathbf{n}}_{A}\right)\right] p_{N \alpha}\right\} \frac{G_{2}^{N}\left(Q_{N}^{2}, Q_{N} \cdot p_{N} / m_{N}\right)}{m_{N}^{3}}\right], \\
& G_{2}^{A}\left(Q^{2}, Q \cdot P_{A} / m_{A}\right) \\
& =-\frac{m_{A}^{2}}{\mathbf{Q}^{2}} \sum_{t_{N}} \int d^{3} p_{N} \frac{m_{N}}{p_{N}^{0}} \int d E\left(Q^{0} \frac{Q_{N}^{0}}{Q^{0}} \frac{1}{n_{A}^{1}} g^{1 \alpha}+\frac{1}{n_{A}^{3}}\left[Q_{N}^{0} g^{3 \alpha}-|\mathrm{Q}| g^{0 \alpha}\right)\right) \\
& \times\left(\mathcal{S}_{\alpha}\left(\mathbf{p}_{N} E t_{N} \widehat{\mathbf{n}}_{A}\right) \frac{G_{1}^{N}\left(Q_{N}^{2}, Q_{N} \cdot p_{N} / m_{N}\right)}{m_{N}}\right. \\
& \left.+\left\{\left(Q_{N} \cdot p_{N}\right) \mathcal{S}_{\alpha}\left(\mathbf{p}_{N} E t_{N} \widehat{\mathbf{n}}_{A}\right)-\left[Q_{N} \cdot \mathcal{S}\left(\mathbf{p}_{N} E t_{N} \widehat{\mathbf{n}}_{A}\right)\right] p_{N \alpha}\right\} \frac{G_{2}^{N}\left(Q_{N}^{2}, Q_{N} \cdot p_{N} / m_{N}\right)}{m_{N}^{3}}\right) .
\end{aligned}
$$

(D3d)

In Eq. (D3) the four-vector $\mathscr{S}\left(\mathbf{p}_{N} E t_{N} \widehat{\mathbf{n}}_{A}\right)$ of Eq. (3.9) is used. The final form of the nuclear spin structure functions in extraction scheme (B) taken for their numerical analysis follows from Eqs. (D3c) and (D3d) as the corresponding results (D2) follow from Eq. (3.11).

With respect to the spin structure functions a third extraction scheme is possible. It is based on the second-rank tensor $\Delta_{\alpha \beta}\left(Q, P_{A}, n_{A}\right)$, which is defined in Eq. (D1b) and which is identified with its PWIA form of Eq. (3.8), i.e., 
$\Delta_{\alpha \beta}\left(Q, P_{A}, n_{A}\right)$

$$
\begin{aligned}
=\sum_{t_{N}} \int d^{3} p_{N} \frac{m_{N}}{p_{N}^{0}} \int d E Q_{N \alpha} & \left(\mathcal{S}_{\beta}\left(\mathbf{p}_{N} E t_{N} \hat{\mathbf{n}}_{A}\right) \frac{G_{1}^{N\left(t_{N}\right)}\left(Q_{N}^{2}, Q_{N} \cdot p_{N} / m_{N}\right)}{m_{N}}\right. \\
& \left.+\left\{\left(Q_{N} \cdot p_{N}\right) \mathcal{S}_{\beta}\left(\mathbf{p}_{N} E t_{N} \hat{\mathbf{n}}_{A}\right)-\left[Q_{N} \cdot \mathcal{S}\left(\mathbf{p}_{N} E t_{N} \hat{\mathbf{n}}_{A}\right)\right] p_{N \beta}\right\} \frac{G_{2}^{N\left(t_{N}\right)}\left(Q_{N}^{2}, Q_{N} \cdot p_{N} / m_{N}\right)}{m_{N}^{3}}\right) .
\end{aligned}
$$

(D4)

The spin structure functions $G_{1}^{A}$ and $G_{2}^{A}$ can be derived from the exact second-rank tensor (D1b) by contractions, i.e., by

$$
\begin{aligned}
& G_{1}^{A}\left(Q^{2}, Q \cdot P_{A} / m_{A}\right)=\frac{m_{A}}{\left(Q \cdot n_{A}\right)} g^{\alpha \beta} \Delta_{\alpha \beta}\left(Q, P_{A}, n_{A}\right), \\
& G_{2}^{A}\left(Q^{2}, Q \cdot P_{A} / m_{A}\right)=-\frac{m_{A}}{Q^{2}\left(Q \cdot n_{A}\right)} Q^{\alpha} P_{A}^{\beta} \Delta_{\alpha \beta}\left(Q, P_{A}, n_{A}\right),
\end{aligned}
$$

and the third extraction scheme applies those results to the approximated form (D4) of that second-rank tensor. We note that some contractions, e.g., Eq. (D5a), use diagonal matrix elements of the tensor which are entirely absent in the combination $i \epsilon^{\mu \nu \alpha \beta} \Delta_{\alpha \beta}\left(Q, P_{A}, n_{A}\right)$ for the nuclear current tensor. Contractions with other tensors, i.e., with $Q^{\alpha} n_{A}^{\beta}, P_{A}^{\alpha} Q^{\beta}, P_{A}^{\alpha} P_{A}^{\beta}$, and so on, yield consistency relations, e.g.,

$$
G_{1}^{A}\left(Q^{2}, Q \cdot P_{A} / m_{A}\right)+\frac{Q \cdot P_{A}}{m_{A}^{2}} G_{2}^{A}\left(Q^{2}, Q \cdot P_{A} / m_{A}\right)=-\frac{m_{A}}{Q^{2}} Q^{\alpha} n_{A}^{\beta} \Delta_{\alpha \beta}\left(Q, P_{A}, n_{A}\right)
$$

That relation holds for the exact form of the second-rank tensor $\Delta_{\alpha \beta}\left(Q, P_{A}, n_{A}\right)$, but it is violated for its approximated form (D4). We declare the convolution formulas for the spin structure functions, resulting from Eqs. (D5a) and (D5b) and given by

$$
\begin{aligned}
G_{1}^{A}\left(Q^{2}, Q \cdot P_{A} / m_{A}\right)=\frac{m_{A}}{m_{N}} \sum_{t_{N}} \int d^{3} p_{N} \frac{m_{N}}{p_{N}^{0}} \int d E & \frac{G_{1}^{N\left(t_{N}\right)}\left(Q_{N}^{2}, Q_{N} \cdot p_{N} / m_{N}\right)}{m_{N}} \\
\times & {\left[m_{N}\left\{f_{1}\left(p_{N} E t_{N}\right)+\left[\left(\hat{\mathbf{p}}_{N} \cdot \hat{\mathbf{Q}}\right)^{2}-\frac{1}{3}\right] f_{2}\left(p_{N} E t_{N}\right)\right\}\right.} \\
& \left.+\left[\left(\hat{\mathbf{p}}_{N} \cdot \hat{\mathbf{Q}}\right)^{2}\left(p_{N}^{0}-m_{N}\right)-Q_{N}^{0} \frac{\mathbf{p}_{N} \cdot \hat{\mathbf{Q}}}{|\mathbf{Q}|}\right]\left[f_{1}\left(p_{N} E t_{N}\right)+\frac{2}{3} f_{2}\left(p_{N} E t_{N}\right)\right]\right],
\end{aligned}
$$

$$
\begin{aligned}
& G_{2}^{A}\left(Q^{2}, Q \cdot P_{A} / m_{A}\right)=\frac{m_{A}^{2}}{m_{N}^{2}} \sum_{t_{N}} \int d^{3} p_{N} \frac{m_{N}}{p_{N}^{0}} \int d E \frac{Q Q_{N}}{Q^{2}} \\
& \times\left[\int G_{1}^{N\left(t_{N}\right)}\left(Q_{N}^{2}, Q_{N} \cdot p_{N} / m_{N}\right) \frac{\mathbf{p}_{N} \cdot \hat{\mathbf{Q}}}{|\mathbf{Q}|}\right. \\
& \left.+G_{2}^{N\left(t_{N}\right)}\left(Q_{N}^{2}, Q_{N} \cdot p_{N} / m_{N}\right)\left(\widehat{\mathbf{p}}_{N} \cdot \hat{\mathbf{Q}}\right)^{2} \frac{p_{N}^{0}-m_{N}}{m_{N}}\right) \\
& \times\left[f_{1}\left(p_{N} E t_{N}\right)+\frac{2}{3} f_{2}\left(p_{N} E t_{N}\right)\right] \\
& \left.+G_{2}^{N\left(t_{N}\right)}\left(Q_{N}^{2}, Q_{N} \cdot p_{N} / m_{N}\right) \frac{p_{N}^{0}}{m_{N}}\left\{f_{1}\left(p_{N} E t_{N}\right)+\left[\left(\widehat{\mathbf{p}}_{N} \cdot \hat{\mathbf{Q}}\right)^{2}-\frac{1}{3}\right] f_{2}\left(p_{N} E t_{N}\right)\right\}\right],
\end{aligned}
$$


extraction scheme (C). That extraction scheme is used in Ref. [13], with the additional approximation $Q_{N}=Q$, which is in this context a minor approximation introducing errors smaller than $1 \%$. We shall also use extraction scheme (C), but according to Eq. (D7) with $Q_{N} \neq Q$ for the purpose of comparison. However, because of its inherent and irreparable inconsistencies we dislike it. In addition, extraction scheme (C) does not allow us to derive the response functions $R_{T L^{\prime}}$ and $R_{T^{\prime}}$ from the tensor $\Delta_{\alpha \beta}\left(Q, P_{A}, n_{A}\right)$ of Eq. (D1b).
[1] C. Marchand et al., Phys. Lett. 153B, 29 (1985).

[2] K. Dow et al., Phys. Rev. Lett. 61, 1706 (1988).

[3] D. Day et al., Phys. Rev. Lett. 43, 1143 (1979).

[4] S. Rock et al., Phys. Rev. C 26, 1592 (1982).

[5] H. Hajduk-Meier, Ch. Hajduk, P. U. Sauer, and W. Theis, Nucl. Phys. A395, 332 (1983).

[6] H. Hajduk-Meier, U. Oelfke, and P. U. Sauer, Nucl. Phys. A499, 637 (1989).

[7] C. Ciofi degli Atti, E. Pace, and G. Salmè, Phys. Lett. 141B, 14 (1984).

[8] R. Schiavilla, V. R. Pandharipande, and R. B. Wiringa, Nucl. Phys. A449, 219 (1986).

[9] H. Hajduk-Meier and P. U. Sauer, Nucl. Phys. A499, 669 (1989).

[10] E. van Meijgaard and J. A. Tjon, Phys. Rev. Lett. 57, 3011 (1986); 61, 1461 (1988); Phys. Rev. C 42, 74 (1990); 42, 96 (1990).

[11] E. W. Otten, Prog. Part. Nucl. Phys. 24, 103 (1990).

[12] T. W. Donnelly and A. S. Raskin, Ann. Phys. (N.Y.) 169, 247 (1986).

[13] B. Blankleider and R. M. Woloshyn, Phys. Rev. C 29, 538
(1984).

[14] M. Lacombe, B. Loiseau, S. M. Richard, R. Vinh Mau, J. Côté, P. Pirès, and R. de Tourreil, Phys. Rev. C 21, 861 (1980).

[15] A. Stadler, P. U. Sauer, and W. Glöckle, Phys. Rev. C 44, 2319 (1991); A. Stadler, private communication.

[16] Ch. Hajduk and P. U. Sauer, Nucl. Phys. A369, 321 (1981).

[17] S. Galster et al., Nucl. Phys. B32, 221 (1971).

[18] C. E. Woodward et al., Phys. Rev. C 44, R571 (1991).

[19] C. E. Woodward et al., Phys. Rev. Lett. 65, 698 (1990).

[20] C. Jones, Ph.D. thesis, California Institute of Technology, 1992 (unpublished).

[21] A. K. Thompson et al., Phys. Rev. Lett. 68, 2901 (1992).

[22] J. Friar, B. F. Gibson, G. L. Payne, A. M. Bernstein, and T. E. Chupp, Phys. Rev. C 42, 2310 (1990).

[23] HERMES Collaboration, Proposal to Measure the SpinDependent Structure Functions of the Neutron and the Proton at HERA, 1990.

[24] J. D. Bjorken and S. D. Drell, Relativistic Quantum Mechanics (McGraw-Hill, New York, 1964). 